\title{
T-coercivity for the Maxwell problem with sign-changing coefficients
}

\author{
Anne-Sophie Bonnet-Ben Dhia ${ }^{1}$, Lucas Chesnel ${ }^{2}$, Patrick Ciarlet Jr. $^{3}$
}

December 6, 2012

\begin{abstract}
In this paper, we study the time-harmonic Maxwell problem with sign-changing permittivity and/or permeability, set in a domain of $\mathbb{R}^{3}$. We prove, using the T-coercivity approach, that the well-posedness of the two canonically associated scalar problems, with Dirichlet and Neumann boundary conditions, implies the well-posedness of the Maxwell problem. This allows us to give simple and sharp criteria, obtained in the study of the scalar cases, to ensure that the Maxwell transmission problem between a classical dielectric material and a negative metamaterial is well-posed.
\end{abstract}

Key words. Maxwell's equations, interface problem, metamaterial, compact imbedding, signchanging coefficients.

\section{Introduction}

We investigate the time-harmonic Maxwell problem in a composite material surrounded by a perfect conductor. A composite material is modeled by non constant electric permittivity $\varepsilon$ and magnetic permeability $\mu$. It is well-known that some materials, like metals at optical frequencies, are almost dissipationless and have a dielectric permittivity whose real part is negative. More surprising is the possibility of realizing materials, called negative metamaterials, which exhibit both negative real valued permittivity and permeability in some appropriate range of frequencies. The association of classical dielectrics and such negative materials has very exciting potential applications such as plasmonic waveguides, perfect lenses [31, 21, 27], photonic traps, subwavelength cavities [16] ... From a mathematical point of view, the change of sign of the coefficients $\varepsilon$ and/or $\mu$ in the medium raises a lot of original questions for the corresponding electromagnetic model, both for the mathematical analysis and the numerical simulation $[26,28,17]$. Indeed, standard theorems proving the well-posedness of the problem and the convergence of conventional numerical methods are no-longer valid in such situations. Consequently, and generally speaking, the questions we have to address are the following. Can we extend the classical theory to configurations with sign-changing coefficients? And if not, is there a new functional framework in which well-posedness and stability properties can be recovered?

For $2 \mathrm{D}$ configurations, the corresponding electromagnetic model reduces to a scalar problem involving the operators $-\operatorname{div}(\sigma \nabla \cdot)$ with Dirichlet or Neumann boundary condition, $\sigma$ being equal to $\varepsilon^{-1}$ or $\mu^{-1}$. Those scalar problems have been thoroughly investigated $[5,35,7,24,2,12,3,10,13]$ and sharp results have been recently obtained using the simple variational technique of the T-coercivity. The problems are proved to be of Fredholm type in the classical functional framework if the contrasts (ratios of the values of $\sigma$ across the interface) are outside some interval, which always contains the value -1 . This interval reduces to $\{-1\}$ if (and only if) the interface is smooth (see also [14, 25] for approaches relying on integral equations). For a contrast equal to -1 , the problems are severely ill-posed (not Fredholm) in $\mathrm{H}^{1}$. The influence of corners in the interface, noticed for instance in $[30,32]$, has been clarified in $[15,8,29]$. When the interface between the dielectric and the negative material has a corner, depending on the value of the contrast in $\sigma$, the scalar problems can be ill-posed (not Fredholm) in $\mathrm{H}^{1}$, even for

\footnotetext{
${ }^{1}$ Laboratoire POEMS, UMR 7231 CNRS/ENSTA/INRIA, ENSTA ParisTech, 828, Boulevard des Maréchaux, 91762 Palaiseau, France; email: Anne-Sophie.Bonnet-Bendhia@ensta-paristech.fr

${ }^{2}$ Laboratoire POEMS, UMR 7231 CNRS/ENSTA/INRIA, ENSTA ParisTech, 828, Boulevard des Maréchaux, 91762 Palaiseau, France; email: Lucas.Chesnel@ensta-paristech.fr

${ }^{3}$ Laboratoire POEMS, UMR 7231 CNRS/ENSTA/INRIA, ENSTA ParisTech, 828, Boulevard des Maréchaux, 91762 Palaiseau, France; email: Patrick.Ciarlet@ensta-paristech.fr
} 
contrasts different from -1 , because of the onset of "strong" singularities at the corner. Well-posedness can be recovered by working in a new functional framework in which a radiation condition at the corner is imposed [4].

For scalar problems, the theory is now quite mature. Now, we wish to obtain such results for Maxwell problems, and first, the results of well-posedness using variational techniques. These variational methods are interesting because they allow one to consider rather general configurations: Lipschitz-continuous interface between the positive and the negative material and $\mathrm{L}^{\infty}$ coefficients $\varepsilon, \varepsilon^{-1}, \mu, \mu^{-1}$. However, it appears that the geometric approach followed for studying the scalar problems is difficult to apply because of the nature of functional spaces used for Maxwell problems. Therefore, we will proceed differently. Again, we will use the T-coercivity technique but in a different form. We will prove that one can construct T-coercivity operators which allow us to restore some coercivity property as soon as the associated 3D scalar problems are well-posed. This will provide very simple criteria (those of the scalar problems) to ensure that Maxwell problems are well-posed. When the contrasts in $\varepsilon$ and/or $\mu$ lie inside the critical intervals, the definition of a new functional framework taking into account the gradients of the strong singularities, is still an open question.

The outline of the paper is the following. The definition of the problem and the notations are introduced in Section 2. In Section 3, we give an equivalent formulation of the problems, using some classical functional spaces for the study of Maxwell problems: $\mathbf{V}_{N}(\varepsilon ; \Omega)$ for the electric field and $\mathbf{V}_{T}(\mu ; \Omega)$ for the magnetic field; some divergence free condition is included in their definition. Section 4 expresses the main idea of the paper: how to build a T-coercivity operator for the Maxwell problems when the associated scalar problems are well-posed. For the sake of clarity, we present it in the canonical cases $\varepsilon=1$ for the electric field and $\mu=1$ for the magnetic field. Then, we use these results and a technique due to [19] to prove some result of compact embedding of $\mathbf{V}_{N}(\varepsilon ; \Omega)$ and $\mathbf{V}_{T}(\mu ; \Omega)$ in $\mathbf{L}^{2}(\Omega):=\mathrm{L}^{2}(\Omega)^{3}$, extending [6]. Again, let us underline that these results are not classical in the literature when the coefficients $\varepsilon$ and $\mu$ change sign. In Section 6, we state the main theorem of this work, summing up the previous results: electric and magnetic Maxwell transmission problems are well-posed as soon as the associated 3D scalar problems are well-posed. We illustrate this result on a series of canonical geometries. Finally, we present some generalizations in Section 8. First, we are interested in configurations where the scalar problems are well-posed in the Fredholm sense with a non trivial kernel. Second, we consider the case of a non-simply connected domain whose boundary is not connected ${ }^{4}$. This study covers the case of non simply connected domains with connected boundary and the case of simply connected domains with non connected boundary.

\section{Setting of the problem}

Let $\Omega$ be a domain in $\mathbb{R}^{3}$, i.e. an open, connected and bounded subset of $\mathbb{R}^{3}$ with a Lipschitz-continuous boundary $\partial \Omega$. For some $\omega \neq 0(\omega \in \mathbb{R})$, the time harmonic Maxwell's equations are given by

$$
\operatorname{curl} \boldsymbol{E}-i \omega \mu \boldsymbol{H}=0 \quad \text { and } \quad \operatorname{curl} \boldsymbol{H}+i \omega \varepsilon \boldsymbol{E}=\boldsymbol{J} \quad \text { in } \Omega \text {. }
$$

Above, $\boldsymbol{E}$ and $\boldsymbol{H}$ are respectively the electric and magnetic components of the electromagnetic field. The source term $\boldsymbol{J}$ is the current density. We suppose that the medium $\Omega$ is surrounded by a perfect conductor and we impose the boundary conditions

$$
\boldsymbol{E} \times \boldsymbol{n}=0 \quad \text { and } \quad \mu \boldsymbol{H} \cdot \boldsymbol{n}=0 \quad \text { on } \partial \Omega,
$$

where $\boldsymbol{n}$ denotes the unit outward normal vector to $\partial \Omega$. We assume that the dielectric permittivity $\varepsilon$ and the magnetic permeability $\mu$ are real valued functions which belong to $\mathrm{L}^{\infty}(\Omega)$, with $\varepsilon^{-1}, \mu^{-1} \in \mathrm{L}^{\infty}(\Omega)$. Let us introduce some classical spaces in the study of Maxwell's equations:

$$
\begin{array}{ll}
\mathbf{L}^{2}(\Omega) & :=\mathrm{L}^{2}(\Omega)^{3} ; \\
\mathbf{H}(\mathbf{c u r l} ; \Omega) & :=\left\{\boldsymbol{u} \in \mathbf{L}^{2}(\Omega) \mid \operatorname{curl} \boldsymbol{u} \in \mathbf{L}^{2}(\Omega)\right\} ; \\
\mathbf{H}_{N}(\mathbf{c u r l} ; \Omega) & :=\{\boldsymbol{u} \in \mathbf{H}(\operatorname{curl} ; \Omega) \mid \boldsymbol{u} \times \boldsymbol{n}=0 \text { on } \partial \Omega\} ; \\
\mathbf{V}_{N}(\xi ; \Omega) & :=\{\boldsymbol{u} \in \mathbf{H}(\operatorname{curl} ; \Omega) \mid \operatorname{div}(\xi \boldsymbol{u})=0, \boldsymbol{u} \times \boldsymbol{n}=0 \text { on } \partial \Omega\}, \text { for } \xi \in \mathrm{L}^{\infty}(\Omega) ; \\
\mathbf{V}_{T}(\xi ; \Omega) & :=\{\boldsymbol{u} \in \mathbf{H}(\operatorname{curl} ; \Omega) \mid \operatorname{div}(\xi \boldsymbol{u})=0, \xi \boldsymbol{u} \cdot \boldsymbol{n}=0 \text { on } \partial \Omega\}, \text { for } \xi \in \mathrm{L}^{\infty}(\Omega) .
\end{array}
$$

\footnotetext{
${ }^{4}$ The Figure 2 at the end of this paper gives an example of such a geometry.
} 
For simplicity, the current density $\boldsymbol{J}$ will be chosen in $\mathbf{L}^{2}(\Omega)$ with $\operatorname{div} \boldsymbol{J}=0^{5}$. We denote indistinctly $(\cdot, \cdot)$ the inner products of $\mathrm{L}^{2}(\Omega)$ and $\mathbf{L}^{2}(\Omega)$ and $\|\cdot\|$ the associated norms. The spaces $\mathbf{H}(\mathbf{c u r l} ; \Omega)$, $\mathbf{H}_{N}(\operatorname{curl} ; \Omega), \mathbf{V}_{N}(\xi ; \Omega)$ and $\mathbf{V}_{T}(\xi ; \Omega)$ are endowed with the inner product

$$
(\cdot, \cdot)_{\operatorname{curl}}=(\cdot, \cdot)+(\operatorname{curl} \cdot, \operatorname{curl} \cdot) .
$$

Classically, we prove that if $(\boldsymbol{E}, \boldsymbol{H})$ satisfies (1)-(2), $\boldsymbol{E}$ and $\boldsymbol{H}$ are respectively solutions to the problems

Find $\boldsymbol{E} \in \mathbf{H}(\mathbf{c u r l} ; \Omega)$ such that:

$$
\begin{array}{lll}
\operatorname{curl} \mu^{-1} \operatorname{curl} \boldsymbol{E}-\omega^{2} \varepsilon \boldsymbol{E} & =i \omega \boldsymbol{J} & \text { in } \Omega \\
\boldsymbol{E} \times \boldsymbol{n} & =0 & \text { on } \partial \Omega
\end{array}
$$

Find $\boldsymbol{H} \in \mathbf{H}($ curl $; \Omega)$ such that:

$$
\begin{aligned}
& \operatorname{curl} \varepsilon^{-1} \operatorname{curl} \boldsymbol{H}-\omega^{2} \mu \boldsymbol{H}=\operatorname{curl} \varepsilon^{-1} \boldsymbol{J} \quad \text { in } \Omega \\
& \begin{array}{llll}
\mu \boldsymbol{H} \cdot \boldsymbol{n} & = & 0 & \text { on } \partial \Omega \\
\varepsilon^{-1}(\operatorname{curl} \boldsymbol{H}-\boldsymbol{J}) \times \boldsymbol{n} & = & 0 & \text { on } \partial \Omega
\end{array}
\end{aligned}
$$

As already announced in the introduction, we want to find criteria for $\varepsilon$ and $\mu$ to ensure that problems (3) and (4) are well-posed in the Fredholm sense. Classically, for the study of Maxwell's equations, our strategy will consist in working in the space $\mathbf{V}_{N}(\varepsilon ; \Omega)$ for the electric field and in the space $\mathbf{V}_{T}(\mu ; \Omega)$ for the magnetic field. Indeed, for example, if $\boldsymbol{E}$ satisfies $(3)$ and if $\omega \neq 0$, then $\operatorname{div}(\varepsilon \boldsymbol{E})=0$, so $\boldsymbol{E}$ belongs to the space $\mathbf{V}_{N}(\varepsilon ; \Omega)$. Therefore, the Fredholm property for the problem (3) will rely on two arguments: first the compact embedding of $\mathbf{V}_{N}(\varepsilon ; \Omega)$ in $\mathbf{L}^{2}(\Omega)$, secondly, the isomorphism property for the principal part $\operatorname{curl} \mu^{-1} \operatorname{curl} \cdot: \mathbf{V}_{N}(\varepsilon ; \Omega) \rightarrow \mathbf{V}_{N}(\varepsilon ; \Omega)^{*}$. In a symmetric way, the Fredholm property for the magnetic field relies on the compact embedding of $\mathbf{V}_{T}(\mu ; \Omega)$ in $\mathbf{L}^{2}(\Omega)$ and on the isomorphism property for the principal part $\operatorname{curl} \varepsilon^{-1} \operatorname{curl} \cdot: \mathbf{V}_{T}(\mu ; \Omega) \rightarrow \mathbf{V}_{T}(\mu ; \Omega)^{*}$.

\section{Equivalent formulations}

Let us first give equivalent formulations to problem (1)-(2) in the spaces $\mathbf{V}_{N}(\varepsilon ; \Omega)$ and $\mathbf{V}_{T}(\mu ; \Omega)$.

\subsection{Problem for the electric field}

Consider $a^{\varepsilon}$ the sesquilinear form such that $a^{\varepsilon}\left(\varphi, \varphi^{\prime}\right)=\left(\varepsilon \nabla \varphi, \nabla \varphi^{\prime}\right)$ for all $\varphi, \varphi^{\prime} \in \mathrm{H}_{0}^{1}(\Omega)$. With the Riesz representation theorem, we define the operator $A^{\varepsilon}: \mathrm{H}_{0}^{1}(\Omega) \rightarrow \mathrm{H}_{0}^{1}(\Omega)$ such that, for all $\varphi, \varphi^{\prime} \in \mathrm{H}_{0}^{1}(\Omega)$, $\left(\nabla\left(A^{\varepsilon} \varphi\right), \nabla \varphi^{\prime}\right)=a^{\varepsilon}\left(\varphi, \varphi^{\prime}\right)$. On $\mathrm{H}_{0}^{1}(\Omega)$, we define the norm $\|\cdot\|_{\mathrm{H}_{0}^{1}(\Omega)}=\|\nabla \cdot\|$. Let $\langle\cdot, \cdot\rangle$ designate the duality pairing $\mathrm{H}^{-1}(\Omega) \times \mathrm{H}_{0}^{1}(\Omega)$. Now, let us introduce an assumption for the $3 \mathrm{D}$ scalar problem related to the electric field. Here, $C>0$ is a constant.

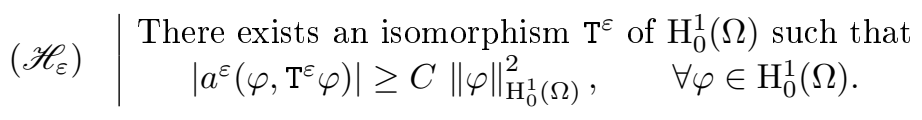

Lemma 3.1 Assumption $\left(\mathscr{H}_{\varepsilon}\right)$ holds true if and only if $A^{\varepsilon}: \mathrm{H}_{0}^{1}(\Omega) \rightarrow \mathrm{H}_{0}^{1}(\Omega)$ is an isomorphism. In this case, for all $f \in \mathrm{H}^{-1}(\Omega)$, there exists a unique solution to the problem

$$
\begin{aligned}
& \text { Find } \varphi \in \mathrm{H}_{0}^{1}(\Omega) \text { such that : } \\
& a^{\varepsilon}\left(\varphi, \varphi^{\prime}\right)=\left\langle f, \varphi^{\prime}\right\rangle, \quad \forall \varphi^{\prime} \in \mathrm{H}_{0}^{1}(\Omega)
\end{aligned}
$$

Besides, there exists a constant $C>0$ independent of $f$ such that $\|\varphi\|_{\mathrm{H}_{0}^{1}(\Omega)} \leq C\|f\|_{\mathrm{H}^{-1}(\Omega)}$.

Remark 3.2 Classically, Eq. (5) can be rewritten $-\operatorname{div}(\varepsilon \nabla \varphi)=f$ in $\mathrm{H}^{-1}(\Omega)$. We will see in section 7 that, in practice, it is possible to build explicitly such operators $T^{\varepsilon}$, depending on the values of $\varepsilon$ and on the geometry of the domain. Actually, assumption $\left(\mathscr{H}_{\varepsilon}\right)$ is an assumption on $\varepsilon$ and on the geometry of the domain only (for more details see [2]).

\footnotetext{
${ }^{5}$ The case $\operatorname{div} \boldsymbol{J} \neq 0$ can be handled similarly with the tools that we propose, see Remark 6.3 below.
} 
Proof. Assume $\left(\mathscr{H}_{\varepsilon}\right)$ to be true. Since $\mathrm{T}^{\varepsilon}: \mathrm{H}_{0}^{1}(\Omega) \rightarrow \mathrm{H}_{0}^{1}(\Omega)$ is an isomorphism, $\varphi$ satisfies $(5)$ if and only if $\varphi$ is a solution of the problem "find $\varphi \in \mathrm{H}_{0}^{1}(\Omega)$ such that $a^{\varepsilon}\left(\varphi, \mathrm{T}^{\varepsilon} \varphi^{\prime}\right)=\left\langle f, \mathrm{~T}^{\varepsilon} \varphi^{\prime}\right\rangle$, for all $\varphi^{\prime} \in \mathrm{H}_{0}^{1}(\Omega)$ ". Lax-Milgram theorem then allows us to conclude that problem (5) is well-posed because $\left(\varphi, \varphi^{\prime}\right) \mapsto a^{\varepsilon}\left(\varphi, \mathrm{T}^{\varepsilon} \varphi^{\prime}\right)$ is coercive on $\mathrm{H}_{0}^{1}(\Omega) \times \mathrm{H}_{0}^{1}(\Omega)$ by assumption. This approach also proves that $A^{\varepsilon}: \mathrm{H}_{0}^{1}(\Omega) \rightarrow \mathrm{H}_{0}^{1}(\Omega)$ is an isomorphism. Reciprocally, if $A^{\varepsilon}: \mathrm{H}_{0}^{1}(\Omega) \rightarrow \mathrm{H}_{0}^{1}(\Omega)$ is an isomorphism, taking $\mathrm{T}^{\varepsilon}=A^{\varepsilon}$, one obtains, for all $\varphi \in \mathrm{H}_{0}^{1}(\Omega),\left|a^{\varepsilon}\left(\varphi, \mathrm{T}^{\varepsilon} \varphi\right)\right|=\left\|A^{\varepsilon} \varphi\right\|_{\mathrm{H}_{0}^{1}(\Omega)}^{2} \geq C\|\varphi\|_{\mathrm{H}_{0}^{1}(\Omega)}^{2}$.

Theorem 3.3 Assume that $\omega \neq 0$.

1) If $(\boldsymbol{E}, \boldsymbol{H})$ satisfies (1)-(2) then $\boldsymbol{E}$ is a solution of the problem

$$
\mid \begin{aligned}
& \text { Find } \boldsymbol{E} \in \mathbf{V}_{N}(\varepsilon ; \Omega) \text { such that for all } \boldsymbol{E}^{\prime} \in \mathbf{V}_{N}(\varepsilon ; \Omega): \\
& \int_{\Omega} \mu^{-1} \operatorname{curl} \boldsymbol{E} \cdot \operatorname{curl} \boldsymbol{E}^{\prime}-\omega^{2} \varepsilon \boldsymbol{E} \cdot \boldsymbol{E}^{\prime}=i \omega \int_{\Omega} \boldsymbol{J} \cdot \boldsymbol{E}^{\prime} .
\end{aligned}
$$

2) Assume $\left(\mathscr{H}_{\varepsilon}\right)$ to be true. If $\boldsymbol{E}$ satisfies (6) then $\left(\boldsymbol{E},(i \omega \mu)^{-1} \mathbf{c u r l} \boldsymbol{E}\right)$ satisfies (1)-(2).

Proof. 1) If $\boldsymbol{E}$ satisfies (1)-(2), then $\boldsymbol{E}$ is a solution of (3). On the other hand, since $\omega \neq 0$, there holds $\operatorname{div}(\varepsilon \boldsymbol{E})=0$. This allows us to show that $\boldsymbol{E}$ satisfies (6).

2) Now, let us prove that if $\boldsymbol{E} \in \mathbf{V}_{N}(\varepsilon ; \Omega) \subset \mathbf{H}_{N}(\operatorname{curl} ; \Omega)$ satisfies (6) then $\boldsymbol{E}$ is a solution to the problem

$$
\begin{aligned}
& \text { Find } \boldsymbol{E} \in \mathbf{H}_{N}(\operatorname{curl} ; \Omega) \text { such that for all } \boldsymbol{E}^{\prime} \in \mathbf{H}_{N}(\operatorname{curl} ; \Omega) \text { : } \\
& \int_{\Omega} \mu^{-1} \operatorname{curl} \boldsymbol{E} \cdot \operatorname{curl} \boldsymbol{E}^{\prime}-\omega^{2} \varepsilon \boldsymbol{E} \cdot \boldsymbol{E}^{\prime}=i \omega \int_{\Omega} \boldsymbol{J} \cdot \boldsymbol{E}^{\prime} .
\end{aligned}
$$

For all $\boldsymbol{E}^{\prime}$ in $\mathbf{H}_{N}(\mathbf{c u r l} ; \Omega)$, Lemma 3.1 indicates that we can build $\varphi \in \mathrm{H}_{0}^{1}(\Omega)$ such that $\operatorname{div}(\varepsilon \nabla \varphi)=$ $\operatorname{div}\left(\varepsilon \boldsymbol{E}^{\prime}\right)$. The element $\boldsymbol{E}^{\prime}-\nabla \varphi$ belongs to $\mathbf{V}_{N}(\varepsilon ; \Omega)$. Taking $\boldsymbol{E}^{\prime}-\nabla \varphi$ as a test-field in (6) and observing that $(\varepsilon \boldsymbol{E}, \nabla \varphi)=0$ and $(\boldsymbol{J}, \nabla \varphi)=0$ (recall that $\operatorname{div} \boldsymbol{J}=0$ ), one obtains

$$
\int_{\Omega} \mu^{-1} \operatorname{curl} \boldsymbol{E} \cdot \operatorname{curl} \boldsymbol{E}^{\prime}-\omega^{2} \varepsilon \boldsymbol{E} \cdot \boldsymbol{E}^{\prime}=i \omega \int_{\Omega} \boldsymbol{J} \cdot \boldsymbol{E}^{\prime} .
$$

But (3) and (7) are equivalents. Therefore, if $\boldsymbol{E}$ satisfies (6) then $\boldsymbol{E}$ is a solution of (3). There just remains to notice that in this case, $\left(\boldsymbol{E},(i \omega \mu)^{-1} \operatorname{curl} \boldsymbol{E}\right)$ satisfies (1)-(2).

With the Riesz representation theorem, let us introduce the bounded operator $\mathscr{A}_{N}(\omega): \mathbf{V}_{N}(\varepsilon ; \Omega) \rightarrow$ $\mathbf{V}_{N}(\varepsilon ; \Omega)$ such that for all $\boldsymbol{E}, \boldsymbol{E}^{\prime} \in \mathbf{V}_{N}(\varepsilon ; \Omega)$,

$$
\left(\mathscr{A}_{N}(\omega) \boldsymbol{E}, \boldsymbol{E}^{\prime}\right)_{\mathbf{c u r l}}=\left(\mu^{-1} \operatorname{curl} \boldsymbol{E}, \operatorname{curl} \boldsymbol{E}^{\prime}\right)-\omega^{2}\left(\varepsilon \boldsymbol{E}, \boldsymbol{E}^{\prime}\right) .
$$

\subsection{Problem for the magnetic field}

For the study of the magnetic field, we introduce the space

$$
\mathrm{H}_{\#}^{1}(\Omega):=\left\{\varphi \in \mathrm{H}^{1}(\Omega) \mid \int_{\Omega} \varphi=0\right\} .
$$

Notice that the only constant in $\mathrm{H}_{\#}^{1}(\Omega)$ is 0 . Using the Rellich's theorem which indicates that the embedding of $\mathrm{H}^{1}(\Omega)$ in $\mathrm{L}^{2}(\Omega)$ is compact and using that $\Omega$ is connected, we prove by contradiction the

Lemma 3.4 The map $\left(\varphi, \varphi^{\prime}\right) \mapsto\left(\nabla \varphi, \nabla \varphi^{\prime}\right)$ defines an inner product on $\mathrm{H}_{\#}^{1}(\Omega)$. The associated norm is denoted $\|\cdot\|_{\mathrm{H}_{\#}^{1}(\Omega)}$.

Consider $a^{\mu}$ the sesquilinear form such that $a^{\mu}\left(\varphi, \varphi^{\prime}\right)=\left(\mu \nabla \varphi, \nabla \varphi^{\prime}\right)$ for all $\varphi, \varphi^{\prime} \in \mathrm{H}_{\#}^{1}(\Omega)$. With the Riesz representation theorem, we define the operator $A^{\mu}: \mathrm{H}_{\#}^{1}(\Omega) \rightarrow \mathrm{H}_{\#}^{1}(\Omega)$ such that, for all $\varphi, \varphi^{\prime} \in \mathrm{H}_{\#}^{1}(\Omega),\left(\nabla\left(A^{\mu} \varphi\right), \nabla \varphi^{\prime}\right)=a^{\mu}\left(\varphi, \varphi^{\prime}\right)$. As for the study of the electric field, let us introduce a property associated with this $3 \mathrm{D}$ scalar problem for the magnetic field. Again, $C>0$ is a constant.

$$
\left(\mathscr{H}_{\mu}\right) \quad \begin{gathered}
\text { There exists an isomorphism } \mathrm{T}^{\mu} \text { of } \mathrm{H}_{\#}^{1}(\Omega) \text { such that } \\
\left|a^{\mu}\left(\varphi, \mathrm{T}^{\mu} \varphi\right)\right| \geq C\|\varphi\|_{\mathrm{H}_{\#}^{1}(\Omega)}^{2}, \quad \forall \varphi \in \mathrm{H}_{\#}^{1}(\Omega) .
\end{gathered}
$$


Lemma 3.5 Assumption $\left(\mathscr{H}_{\mu}\right)$ holds true if and only if $A^{\mu}: \mathrm{H}_{\#}^{1}(\Omega) \rightarrow \mathrm{H}_{\#}^{1}(\Omega)$ is an isomorphism. In this case, for all $\ell \in\left(\mathrm{H}_{\#}^{1}(\Omega)\right)^{\prime}$, there exists a unique solution to the problem

$$
\mid \begin{aligned}
& \text { Find } \varphi \in \mathrm{H}_{\#}^{1}(\Omega) \text { such that: } \\
& a^{\mu}\left(\varphi, \varphi^{\prime}\right)=\ell\left(\varphi^{\prime}\right), \quad \forall \varphi^{\prime} \in \mathrm{H}_{\#}^{1}(\Omega) .
\end{aligned}
$$

Besides, there exists a constant $C>0$ independent of $\ell$ such that $\|\varphi\|_{\mathrm{H}_{\#}^{1}(\Omega)} \leq C\|\ell\|_{\left(\mathrm{H}_{\#}^{1}(\Omega)\right)^{\prime}}$.

Remark 3.6 Classically, for $f \in \mathrm{L}^{2}(\Omega)$ such that $\int_{\Omega} f=0$, if one defines $\ell$ by $\ell\left(\varphi^{\prime}\right)=\int_{\Omega} f \varphi^{\prime}$ for all $\varphi^{\prime} \in \mathrm{H}_{\#}^{1}(\Omega)$, Eq. (9) can be rewritten $-\operatorname{div}(\mu \nabla \varphi)=f$ in $\mathrm{L}^{2}(\Omega)$ and $\mu \partial_{n} \varphi=0$ in $\mathrm{H}^{-1 / 2}(\partial \Omega)$. As in the electric case, assumption $\left(\mathscr{H}_{\mu}\right)$ is an assumption on $\mu$ and on the geometry of the domain only.

Proof. Assume $\left(\mathscr{H}_{\mu}\right)$ to be true. Since $\mathrm{T}^{\mu}: \mathrm{H}_{\#}^{1}(\Omega) \rightarrow \mathrm{H}_{\#}^{1}(\Omega)$ is an isomorphism, $\varphi$ satisfies (9) if and only if $\varphi$ is a solution to the problem "find $\varphi \in \mathrm{H}_{\#}^{1}(\Omega)$ such that $a^{\mu}\left(\varphi, \mathrm{T}^{\mu} \varphi^{\prime}\right)=\ell\left(\mathrm{T}^{\mu} \varphi^{\prime}\right)$, for all $\varphi^{\prime} \in \mathrm{H}_{\#}^{1}(\Omega)$ ". But Lemma 3.4 ensures that $\left(\varphi, \varphi^{\prime}\right) \mapsto a^{\mu}\left(\varphi, \mathrm{T}^{\varepsilon} \varphi^{\prime}\right)$ is coercive on $\mathrm{H}_{\#}^{1}(\Omega) \times \mathrm{H}_{\#}^{1}(\Omega)$. Then, one can conclude that problem (9) is well-posed with Lax-Milgram theorem. In the same way, we prove that $A^{\mu}: \mathrm{H}_{\#}^{1}(\Omega) \rightarrow \mathrm{H}_{\#}^{1}(\Omega)$ is an isomorphism. Reciprocally, if $A^{\mu}$ is an isomorphism of $\mathrm{H}_{\#}^{1}(\Omega)$, taking $\mathrm{T}^{\mu}=A^{\mu}$, one finds, for $\varphi \in \mathrm{H}_{\#}^{1}(\Omega),\left|a^{\mu}\left(\varphi, \mathrm{T}^{\mu} \varphi\right)\right|=\left\|A^{\mu} \varphi\right\|_{\mathrm{H}_{\#}^{1}(\Omega)}^{2} \geq C\|\varphi\|_{\mathrm{H}_{\#}^{1}(\Omega)}^{2}$.

Adapting the proof of Theorem 3.3, one obtains the

Theorem 3.7 Assume that $\omega \neq 0$.

1) If $(\boldsymbol{E}, \boldsymbol{H})$ satisfies (1)-(2) then $\boldsymbol{H}$ is a solution of the problem

$$
\begin{array}{|l}
\text { Find } \boldsymbol{H} \in \mathbf{V}_{T}(\mu ; \Omega) \text { such that for all } \boldsymbol{H}^{\prime} \in \mathbf{V}_{T}(\mu ; \Omega) \text { : } \\
\int_{\Omega} \varepsilon^{-1} \operatorname{curl} \boldsymbol{H} \cdot \operatorname{curl} \boldsymbol{H}^{\prime}-\omega^{2} \mu \boldsymbol{H} \cdot \boldsymbol{H}^{\prime}=\int_{\Omega} \varepsilon^{-1} \boldsymbol{J} \cdot \operatorname{curl} \boldsymbol{H}^{\prime} .
\end{array}
$$

2) Assume $\left(\mathscr{H}_{\mu}\right)$ to be true. If $\boldsymbol{H}$ satisfies (10) then $\left(i(\omega \varepsilon)^{-1}(\operatorname{curl} \boldsymbol{H}-\boldsymbol{J}), \boldsymbol{H}\right)$ satisfies (1)-(2).

With the Riesz representation theorem, let us introduce the bounded operator $\mathscr{A}_{T}(\omega): \mathbf{V}_{T}(\mu ; \Omega) \rightarrow$ $\mathbf{V}_{T}(\mu ; \Omega)$ such that for all $\boldsymbol{H}, \boldsymbol{H}^{\prime} \in \mathbf{V}_{T}(\mu ; \Omega)$,

$$
\left(\mathscr{A}_{T}(\omega) \boldsymbol{H}, \boldsymbol{H}^{\prime}\right)_{\text {curl }}=\left(\varepsilon^{-1} \operatorname{curl} \boldsymbol{H}, \operatorname{curl} \boldsymbol{H}^{\prime}\right)-\omega^{2}\left(\mu \boldsymbol{H}, \boldsymbol{H}^{\prime}\right) .
$$

\section{The T-coercivity approach in $\mathbf{V}_{N}(1 ; \Omega)$ and in $\mathbf{V}_{T}(1 ; \Omega)$}

For the sake of clarity, we present first the T-coercivity approach for the Maxwell's equations on the simpler cases: $\varepsilon=1$ and $\mu$ possibly sign-changing for the electric field; $\mu=1$ and $\varepsilon$ possibly sign-changing for the magnetic field. This study will be useful for two reasons. On the one hand, the results obtained will serve to prove the compact embeddings of $\mathbf{V}_{N}(\varepsilon ; \Omega)$ and $\mathbf{V}_{T}(\mu ; \Omega)$ in $\mathbf{L}^{2}(\Omega)$. On the other hand, this will give us an insight of how to proceed to study the operators $\mathscr{A}_{N}(0)$ and $\mathscr{A}_{T}(0)$.

Let us recall some classical properties for the spaces $\mathbf{V}_{N}(1 ; \Omega)$ and $\mathbf{V}_{T}(1 ; \Omega)$ (cf. [33, 1]).

- The embeddings of $\mathbf{V}_{N}(1 ; \Omega)$ in $\mathbf{L}^{2}(\Omega)$ and of $\mathbf{V}_{T}(1 ; \Omega)$ in $\mathbf{L}^{2}(\Omega)$ are compact.

- Furthermore, when $\partial \Omega$ is connected (respectively when $\Omega$ is simply connected), the map $(\boldsymbol{u}, \boldsymbol{v}) \mapsto$ $(\operatorname{curl} \boldsymbol{u}, \operatorname{curl} \boldsymbol{v})$ defines an inner product on $\mathbf{V}_{N}(1 ; \Omega)\left(\right.$ resp. on $\mathbf{V}_{T}(1 ; \Omega)$ ) and the associated norm is equivalent to the canonical norm $\boldsymbol{u} \mapsto(\boldsymbol{u}, \boldsymbol{u})_{\text {curl }}^{1 / 2}$.

\subsection{Study for the electric field with a permittivity $\varepsilon=1$}

Let us first study the problem for the electric field.

Lemma 4.1 Let $\Omega$ be a simply connected domain such that $\partial \Omega$ is connected. Assume $\left(\mathscr{H}_{\mu}\right)$ to be true. Then, there exists an isomorphism $\mathbb{T}$ of $\mathbf{V}_{N}(1 ; \Omega)$ such that for all $\boldsymbol{u}, \boldsymbol{v} \in \mathbf{V}_{N}(1 ; \Omega)$,

$$
\left(\mu^{-1} \operatorname{curl} \boldsymbol{u}, \operatorname{curl} \mathbb{T} \boldsymbol{v}\right)=\left(\mu^{-1} \operatorname{curl} \mathbb{T} \boldsymbol{u}, \operatorname{curl} \boldsymbol{v}\right)=(\operatorname{curl} \boldsymbol{u}, \operatorname{curl} \boldsymbol{v}) .
$$


Remark 4.2 One can prove a "reciprocal" assertion. Namely, "there exists an isomorphism $\mathbb{T}$ of $\mathbf{V}_{N}(1 ; \Omega)$ such that $\left(\mu^{-1} \operatorname{curl} \boldsymbol{u}, \operatorname{curl} \mathbb{T} \boldsymbol{u}\right) \geq\|\operatorname{curl} \boldsymbol{u}\|^{2}$, for all $\boldsymbol{u} \in \mathbf{V}_{N}(1 ; \Omega)$ " implies " $\left(\mathscr{H}_{\mu}\right)$ is true".

Proof. $\star$ Definition of $\mathbb{T}$. Consider $\boldsymbol{v} \in \mathbf{V}_{N}(1 ; \Omega)$.

i) Define $\varphi$ the unique element of $H_{\#}^{1}(\Omega)$ such that

$$
\int_{\Omega} \mu \nabla \varphi \cdot \nabla \varphi^{\prime}=\int_{\Omega} \mu \operatorname{curl} \boldsymbol{v} \cdot \nabla \varphi^{\prime}, \quad \forall \varphi^{\prime} \in \mathrm{H}_{\#}^{1}(\Omega)
$$

The function $\varphi$ is well-defined since we have assumed $\left(\mathscr{H}_{\mu}\right)$ to be true.

ii) Observe next that $\mu(\operatorname{curl} \boldsymbol{v}-\nabla \varphi)$ is a divergence free element of $\mathbf{L}^{2}(\Omega)$ such that $\mu(\operatorname{curl} \boldsymbol{v}-\nabla \varphi) \cdot \boldsymbol{n}=0$ on $\partial \Omega$. Since $\Omega$ is simply connected and since $\partial \Omega$ is connected, according to theorem 3.17 in [1] (see also theorem 3.6 in [18]), there exists a unique potential $\mathbb{T} \boldsymbol{v} \in \mathbf{V}_{N}(1 ; \Omega)$ such that $\operatorname{curl} \mathbb{T} \boldsymbol{v}=\mu(\operatorname{curl} \boldsymbol{v}-\nabla \varphi)$. This defines a bounded operator $\mathbb{T}: \mathbf{V}_{N}(1 ; \Omega) \rightarrow \mathbf{V}_{N}(1 ; \Omega)$.

$\star$ Positiveness property. For all $\boldsymbol{u}, \boldsymbol{v} \in \mathbf{V}_{N}(1 ; \Omega)$, we then compute

$$
\left(\mu^{-1} \operatorname{curl} \boldsymbol{u}, \operatorname{curl} \mathbb{T} \boldsymbol{v}\right)=\left(\mu^{-1} \operatorname{curl} \boldsymbol{u}, \mu(\operatorname{curl} \boldsymbol{v}-\nabla \varphi)\right)=(\operatorname{curl} \boldsymbol{u}, \operatorname{curl} \boldsymbol{v}),
$$

because $\boldsymbol{u} \times \boldsymbol{n}=0$ on $\partial \Omega$. Notice we have also $\left(\mu^{-1} \operatorname{curl} \mathbb{T} \boldsymbol{u}, \operatorname{curl} \boldsymbol{v}\right)=(\operatorname{curl} \boldsymbol{u}, \operatorname{curl} \boldsymbol{v})$.

$\star \mathbb{T}$ is an isomorphism of $\mathbf{V}_{N}(1 ; \Omega)$. Let us consider the operator for the electric field $\mathscr{A}_{N}(\omega)$ : $\mathbf{V}_{N}(1 ; \Omega) \rightarrow \mathbf{V}_{N}(1 ; \Omega)$ defined in (8). The previous identity allows us to write

$$
\left(\mathscr{A}_{N}(0)(\mathbb{T} \boldsymbol{u}), \boldsymbol{v}\right)_{\operatorname{curl}}=\left(\mu^{-1} \operatorname{curl} \mathbb{T} \boldsymbol{u}, \operatorname{curl} \boldsymbol{v}\right)=(\operatorname{curl} \boldsymbol{u}, \operatorname{curl} \boldsymbol{v}), \forall \boldsymbol{u}, \boldsymbol{v} \in \mathbf{V}_{N}(1 ; \Omega)
$$

Since $\partial \Omega$ is connected, the map $(\boldsymbol{u}, \boldsymbol{v}) \mapsto(\operatorname{curl} \boldsymbol{u}, \operatorname{curl} \boldsymbol{v})$ defines an inner product on $\mathbf{V}_{N}(1 ; \Omega)$. Consequently, the operator $\mathscr{A}_{N}(0) \circ \mathbb{T}$ is an isomorphism of $\mathbf{V}_{N}(1 ; \Omega)$. Since $\mathscr{A}_{N}(0)$ is selfadjoint, we deduce that $\mathscr{A}_{N}(0)$ and $\mathbb{T}$ are isomorphisms.

We deduce the

Proposition 4.3 Let $\Omega$ be a simply connected domain such that $\partial \Omega$ is connected. Assume $\left(\mathscr{H}_{\mu}\right)$ to be true and $\varepsilon=1$. Then, the problem for the electric field (6) has one and only one solution which continuously depends on the data $\boldsymbol{J}$, for all $\omega \in \mathbb{C} \backslash \mathscr{S}$, where $\mathscr{S}$ is a discrete set.

Proof. According to Lemma 4.1, the operator $\mathscr{A}_{N}(0)$, with $\varepsilon=1$, is an isomorphism when assumption $\left(\mathscr{H}_{\mu}\right)$ holds true. Since $\mathbf{V}_{N}(1 ; \Omega)$ is compactly embedded in $\mathbf{L}^{2}(\Omega), \mathscr{A}_{N}(\omega)-\mathscr{A}_{N}(0)$ is a compact operator of $\mathbf{V}_{N}(1 ; \Omega)$ for all $\omega \in \mathbb{C}$. The analytic Fredholm theorem allows us to conclude.

Remark 4.4 The analysis presented in this paragraph also holds in the case where the assumption " $\varepsilon=1$ " is replaced by the assumption " $\varepsilon \geq C>0$ ". To deal with the case of a sign changing coefficient $\varepsilon$, we will have to assume in addition that $\left(\mathscr{H}_{\varepsilon}\right)$ holds true to be able to build an operator $\mathbb{T}$ with values in $\mathbf{V}_{N}(\varepsilon ; \Omega)$ and to prove that the embedding of $\mathbf{V}_{N}(\varepsilon ; \Omega)$ in $\mathbf{L}^{2}(\Omega)$ is compact.

\subsection{Study for the magnetic field with a permeability $\mu=1$}

Now, let us study the problem for the magnetic field.

Lemma 4.5 Let $\Omega$ be a simply connected domain such that $\partial \Omega$ is connected. Assume $\left(\mathscr{H}_{\varepsilon}\right)$ to be true. Then, there exists an isomorphism $\mathbb{T}$ of $\mathbf{V}_{T}(1 ; \Omega)$ such that for all $\boldsymbol{u}, \boldsymbol{v} \in \mathbf{V}_{T}(1 ; \Omega)$,

$$
\left(\varepsilon^{-1} \operatorname{curl} \boldsymbol{u}, \operatorname{curl} \mathbb{T} \boldsymbol{v}\right)=\left(\varepsilon^{-1} \operatorname{curl} \mathbb{T} u, \operatorname{curl} v\right)=(\operatorname{curl} u, \operatorname{curl} v) .
$$

Remark 4.6 One can again prove a "reciprocal" assertion. Namely, "there exists an isomorphism $\mathbb{T}$ of $\mathbf{V}_{T}(1 ; \Omega)$ such that $\left(\varepsilon^{-1} \operatorname{curl} \boldsymbol{u}, \mathbf{c u r l} \mathbb{T} \boldsymbol{u}\right) \geq\|\operatorname{curl} \boldsymbol{u}\|^{2}$, for all $\boldsymbol{u} \in \mathbf{V}_{T}(1 ; \Omega)$ " implies "( $\left.\mathscr{H}_{\varepsilon}\right)$ is true".

Proof. The proof is very similar to the proof of Lemma 4.1, the boundary conditions being the only changing elements.

$\star$ Definition of $\mathbb{T}$. Consider $\boldsymbol{v} \in \mathbf{V}_{T}(1 ; \Omega)$.

i) First define $\varphi$ the unique element of $\mathrm{H}_{0}^{1}(\Omega)$ such that

$$
\int_{\Omega} \varepsilon \nabla \varphi \cdot \nabla \varphi^{\prime}=\int_{\Omega} \varepsilon \operatorname{curl} \boldsymbol{v} \cdot \nabla \varphi^{\prime}, \quad \forall \varphi^{\prime} \in \mathrm{H}_{0}^{1}(\Omega) .
$$


The function $\varphi$ is well-defined since we have assumed $\left(\mathscr{H}_{\varepsilon}\right)$ to be true.

ii) Then, notice that $\varepsilon(\operatorname{curl} \boldsymbol{v}-\nabla \varphi)$ is a divergence free element of $\mathbf{L}^{2}(\Omega)$. Since $\Omega$ is simply connected and since $\partial \Omega$ is connected, according to theorem 3.12 in [1], there exists a unique potential $\mathbb{T} \boldsymbol{v} \in \mathbf{V}_{T}(1 ; \Omega)$ such that $\operatorname{curl} \mathbb{T} \boldsymbol{v}=\varepsilon(\operatorname{curl} \boldsymbol{v}-\nabla \varphi)$. This defines an operator $\mathbb{T}$ from $\mathbf{V}_{T}(1 ; \Omega)$ to $\mathbf{V}_{T}(1 ; \Omega)$.

$\star$ Positiveness property. For all $\boldsymbol{u}, \boldsymbol{v} \in \mathbf{V}_{T}(1 ; \Omega)$, we then compute

$$
\left(\varepsilon^{-1} \operatorname{curl} \boldsymbol{u}, \operatorname{curl} \mathbb{T} \boldsymbol{v}\right)=\left(\varepsilon^{-1} \operatorname{curl} \boldsymbol{u}, \mu(\operatorname{curl} \boldsymbol{v}-\nabla \varphi)\right)=(\operatorname{curl} \boldsymbol{u}, \operatorname{curl} \boldsymbol{v}),
$$

because $\varphi=0$ on $\partial \Omega$. We have also $\left(\varepsilon^{-1} \operatorname{curl} \mathbb{T} \boldsymbol{u}, \operatorname{curl} \boldsymbol{v}\right)=(\operatorname{curl} \boldsymbol{u}, \operatorname{curl} \boldsymbol{v})$.

$\star \mathbb{T}$ is an isomorphism of $\mathbf{V}_{T}(1 ; \Omega)$. Let us consider the operator for the magnetic field $\mathscr{A}_{T}(\omega)$ : $\mathbf{V}_{T}(1 ; \Omega) \rightarrow \mathbf{V}_{T}(1 ; \Omega)$ defined in (11). The previous identity allows us to write

$$
\left(\mathscr{A}_{T}(0)(\mathbb{T} \boldsymbol{u}), \boldsymbol{v}\right)_{\operatorname{curl}}=\left(\varepsilon^{-1} \operatorname{curl} \mathbb{T} \boldsymbol{u}, \operatorname{curl} \boldsymbol{v}\right)=(\operatorname{curl} \boldsymbol{u}, \operatorname{curl} \boldsymbol{v}), \forall \boldsymbol{u}, \boldsymbol{v} \in \mathbf{V}_{T}(1 ; \Omega) .
$$

Since $\Omega$ is simply connected, the map $(\boldsymbol{u}, \boldsymbol{v}) \mapsto(\operatorname{curl} \boldsymbol{u}, \operatorname{curl} \boldsymbol{v})$ defines an inner product on $\mathbf{V}_{T}(1 ; \Omega)$. Thus, the operator $\mathscr{A}_{T}(0) \circ \mathbb{T}$ is an isomorphism of $\mathbf{V}_{T}(1 ; \Omega)$. Since $\mathscr{A}_{T}(0)$ is selfadjoint, we deduce that $\mathscr{A}_{T}(0)$ and $\mathbb{T}$ are isomorphisms.

Proceeding as in Proposition 4.3, one proves the

Proposition 4.7 Let $\Omega$ be a simply connected domain such that $\partial \Omega$ is connected. Assume ( $\left.\mathscr{H}_{\varepsilon}\right)$ to be true and $\mu=1$. Then, the problem for the magnetic field (10) has one and only one solution which continuously depends on the data $\boldsymbol{J}$, for all $\omega \in \mathbb{C} \backslash \mathscr{S}$, where $\mathscr{S}$ is a discrete set.

Remark 4.8 Like in Remark 4.4, let us indicate that the analysis of this paragraph also holds in the case where the assumption " $\mu=1$ " is replaced by the assumption " $\mu \geq C>0$ ". To deal with the case of a sign changing coefficient $\mu$, we will have furthermore to assume that $\left(\mathscr{H}_{\mu}\right)$ holds true to be able to build an operator $\mathbb{T}$ with values in $\mathbf{V}_{T}(\mu ; \Omega)$ and to prove that the embedding of $\mathbf{V}_{T}(\mu ; \Omega)$ in $\mathbf{L}^{2}(\Omega)$ is compact.

\section{Compactness results}

Define the spaces, for $\xi \in \mathrm{L}^{\infty}(\Omega)$,

$$
\begin{aligned}
& \mathbf{X}_{N}(\xi ; \Omega):=\left\{\boldsymbol{u} \in \mathbf{H}(\operatorname{curl} ; \Omega) \mid \operatorname{div}(\xi \boldsymbol{u}) \in \mathbf{L}^{2}(\Omega), \boldsymbol{u} \times \boldsymbol{n}=0 \text { on } \partial \Omega\right\} \\
& \mathbf{X}_{T}(\xi ; \Omega):=\left\{\boldsymbol{u} \in \mathbf{H}(\operatorname{curl} ; \Omega) \mid \operatorname{div}(\xi \boldsymbol{u}) \in \mathbf{L}^{2}(\Omega), \xi \boldsymbol{u} \cdot \boldsymbol{n}=0 \text { on } \partial \Omega\right\} .
\end{aligned}
$$

Theses spaces are equipped with the norm $\boldsymbol{u} \mapsto\left(\|\boldsymbol{u}\|^{2}+\|\operatorname{div}(\xi \boldsymbol{u})\|^{2}+\|\operatorname{curl} \boldsymbol{u}\|^{2}\right)^{1 / 2}$. In this paragraph, we prove that $\mathbf{X}_{N}(\varepsilon ; \Omega)$ and $\mathbf{X}_{T}(\mu ; \Omega)$ are compactly embedded in $\mathbf{L}^{2}(\Omega)$ when $\left(\mathscr{H}_{\varepsilon}\right)$ and $\left(\mathscr{H}_{\mu}\right)$ hold true, extending the classical theorems of $[33,19,23]$ (for another approach, based on the study of the regularity of fields, in $2 \mathrm{D}$, when $\varepsilon, \mu$ change sign, see [11]). This constitutes a more general result than the one we actually need for our study, namely the compact embedding of $\mathbf{V}_{N}(\varepsilon ; \Omega)$ and $\mathbf{V}_{T}(\mu ; \Omega)$ in $\mathbf{L}^{2}(\Omega)$.

\subsection{Compact embedding of $\mathbf{X}_{N}(\varepsilon ; \Omega)$ in $\mathbf{L}^{2}(\Omega)$}

Let us start by studying the space of electric fields.

Theorem 5.1 Let $\Omega$ be a simply connected domain such that $\partial \Omega$ is connected. Assume ( $\left.\mathscr{H}_{\varepsilon}\right)$ to be true. Then the embedding of $\mathbf{X}_{N}(\varepsilon ; \Omega)$ in $\mathbf{L}^{2}(\Omega)$ is compact.

Proof. Let $\left(\boldsymbol{u}_{n}\right)$ be a bounded sequence of $\mathbf{X}_{N}(\varepsilon ; \Omega)$. Define $f_{n}:=\operatorname{div}\left(\varepsilon \boldsymbol{u}_{n}\right)$ and $\boldsymbol{F}_{n}:=\operatorname{curl} \boldsymbol{u}_{n}$. The sequences $\left(f_{n}\right)$ and $\left(\boldsymbol{F}_{n}\right)$ are respectively bounded in $\mathrm{L}^{2}(\Omega)$ and in $\mathbf{L}^{2}(\Omega)$. Since $\left(\mathscr{H}_{\varepsilon}\right)$ is true, according to Lemma 3.1, there exists, for all $n \in \mathbb{N}, \varphi_{n} \in \mathrm{H}_{0}^{1}(\Omega)$ such that $\operatorname{div}\left(\varepsilon \nabla \varphi_{n}\right)=\operatorname{div}\left(\varepsilon \boldsymbol{u}_{n}\right)$. Then, we notice that $\varepsilon\left(\boldsymbol{u}_{n}-\nabla \varphi_{n}\right)$ is a divergence free element of $\mathbf{L}^{2}(\Omega)$. Since $\partial \Omega$ is connected, there exists (see [1], theorem 3.12) $\boldsymbol{w}_{n} \in \mathbf{V}_{T}(1 ; \Omega)$ such that $\operatorname{curl} \boldsymbol{w}_{n}=\varepsilon\left(\boldsymbol{u}_{n}-\nabla \varphi_{n}\right)$. Thus, for all $n \in \mathbb{N}$, one has $\boldsymbol{u}_{n}=\nabla \varphi_{n}+\varepsilon^{-1} \operatorname{curl} \boldsymbol{w}_{n}$. Let us show now we can extract sequences from $\left(\nabla \varphi_{n}\right)$ and $\left(\mathbf{c u r l} \boldsymbol{w}_{n}\right)$ which converge in $\mathbf{L}^{2}(\Omega)$. 
Lemma 3.1 ensures that $\left(\varphi_{n}\right)$ remains bounded in $\mathrm{H}_{0}^{1}(\Omega)$. The operator $\mathrm{T}^{\varepsilon}$ of hypothesis $\left(\mathscr{H}_{\varepsilon}\right)$ is continuous from $\mathrm{H}_{0}^{1}(\Omega)$ to $\mathrm{H}_{0}^{1}(\Omega)$, $\left(\mathrm{T}^{\varepsilon} \varphi_{n}\right)$ is also bounded in $\mathrm{H}_{0}^{1}(\Omega)$. But $\mathrm{H}_{0}^{1}(\Omega)$ is compactly embedded in $\mathrm{L}^{2}(\Omega)$. Therefore, we can extract a subsequence from $\left(\varphi_{n}\right)$ (still denoted $\left(\varphi_{n}\right)$ ) such that $\left(\mathrm{T}^{\varepsilon} \varphi_{n}\right)$ converges in $\mathrm{L}^{2}(\Omega)$. Introduce $\varphi_{n m}=\varphi_{n}-\varphi_{m}$ and $f_{n m}=f_{n}-f_{m}$. By linearity, there holds: $-\left(\varepsilon \nabla \varphi_{n m}, \nabla \varphi^{\prime}\right)=\left(f_{n m}, \varphi^{\prime}\right)$, for all $\varphi^{\prime} \in \mathrm{H}_{0}^{1}(\Omega)$. Taking $\varphi^{\prime}=\mathrm{T}^{\varepsilon} \varphi_{n m}$, one obtains

$$
C\left\|\varphi_{n m}\right\|_{\mathrm{H}_{0}^{1}(\Omega)}^{2} \leq\left|\left(\varepsilon \nabla \varphi_{n m}, \nabla\left(\mathrm{T}^{\varepsilon} \varphi_{n m}\right)\right)\right|=\left|\left(f_{n m}, \mathrm{~T}^{\varepsilon} \varphi_{n m}\right)\right| .
$$

This shows that $\left(\nabla \varphi_{n}\right)$ is a Cauchy sequence in $\mathbf{L}^{2}(\Omega)$, and so, that it converges.

Now, let us work on the sequence $\left(\operatorname{curl} \boldsymbol{w}_{n}\right)$. We know that $\boldsymbol{w} \mapsto\|\operatorname{curl} \boldsymbol{w}\|$ defines a norm on $\mathbf{V}_{T}(1 ; \Omega)$. Consequently, the sequence $\left(\boldsymbol{w}_{n}\right)$ is bounded in $\mathbf{V}_{T}(1 ; \Omega)$. By the compact imbedding of $\mathbf{V}_{T}(1 ; \Omega)$ in $\mathbf{L}^{2}(\Omega)$, we can extract a subsequence, still denoted $\left(\boldsymbol{w}_{n}\right)$, which converges in $\mathbf{L}^{2}(\Omega)$. According to Lemma 4.5 , there exists an isomorphism $\mathbb{T}$ of $\mathbf{V}_{T}(1 ; \Omega)$ such that

$$
\left|\left(\varepsilon^{-1} \operatorname{curl} \boldsymbol{w}, \operatorname{curl} \mathbb{T} \boldsymbol{w}\right)\right|=\|\operatorname{curl} \boldsymbol{w}\|^{2}, \quad \forall \boldsymbol{w} \in \mathbf{V}_{T}(1 ; \Omega) .
$$

Since $\mathbb{T}$ is continuous, the sequence $\left(\mathbb{T} \boldsymbol{w}_{n}\right)$ is bounded in $\mathbf{V}_{T}(1 ; \Omega)$. So, we can extract a subsequence from $\left(\boldsymbol{w}_{n}\right)$, still denoted $\left(\boldsymbol{w}_{n}\right)$, such that $\left(\mathbb{T} \boldsymbol{w}_{n}\right)$ converges in $\mathbf{L}^{2}(\Omega)$. Since $\operatorname{curl} \varepsilon^{-1} \operatorname{curl} \boldsymbol{w}_{n}=\boldsymbol{F}_{n}$ in $\Omega$ and $\left(\varepsilon^{-1} \operatorname{curl} \boldsymbol{w}_{n}\right) \times \boldsymbol{n}=0$ on $\partial \Omega$, one has $\left(\varepsilon^{-1} \operatorname{curl} \boldsymbol{w}_{n m}, \operatorname{curl} \boldsymbol{w}^{\prime}\right)=\left(\boldsymbol{F}_{n m}, \boldsymbol{w}^{\prime}\right)$, for all $\boldsymbol{w}^{\prime} \in \mathbf{V}_{T}(1 ; \Omega)$ with $\boldsymbol{w}_{n m}=\boldsymbol{w}_{n}-\boldsymbol{w}_{m}$ and $\boldsymbol{F}_{n m}=\boldsymbol{F}_{n}-\boldsymbol{F}_{m}$. Testing with $\boldsymbol{w}^{\prime}=\mathbb{T} \boldsymbol{w}_{n m}$ leads to:

$$
\left\|\operatorname{curl} \boldsymbol{w}_{n m}\right\|^{2}=\left|\left(\boldsymbol{F}_{n m}, \mathbb{T} \boldsymbol{w}_{n m}\right)\right| .
$$

This estimate proves that ( $\left.\operatorname{curl} \boldsymbol{w}_{n}\right)$ is a Cauchy sequence in $\mathbf{L}^{2}(\Omega)$. Consequently, it converges.

Corollary 5.2 Let $\Omega$ be a simply connected domain such that $\partial \Omega$ is connected. Assume ( $\left.\mathscr{H}_{\varepsilon}\right)$ to be true. Then, there exists a constant $C$ such that

$$
\|\boldsymbol{u}\|^{2} \leq C\|\operatorname{curl} \boldsymbol{u}\|^{2}, \quad \forall \boldsymbol{u} \in \mathbf{V}_{N}(\varepsilon ; \Omega) .
$$

Thus, the map $(\boldsymbol{u}, \boldsymbol{v}) \mapsto(\operatorname{curl} \boldsymbol{u}, \operatorname{curl} \boldsymbol{v})$ defines an inner product on $\mathbf{V}_{N}(\varepsilon ; \Omega)$ and the associated norm is equivalent to the canonical norm $\boldsymbol{u} \mapsto(\boldsymbol{u}, \boldsymbol{u})_{\text {curl }}^{1 / 2}$.

Proof. To prove this corollary, it is sufficient to show (12). Let us proceed by contradiction assuming there exists a sequence $\left(\boldsymbol{u}_{n}\right)$ of elements of $\mathbf{V}_{N}(\varepsilon ; \Omega)$ such that

$$
\forall n \in \mathbb{N},\left\|\boldsymbol{u}_{n}\right\|=1 \quad \text { and } \quad \lim _{n \rightarrow \infty}\left\|\operatorname{curl} \boldsymbol{u}_{n}\right\|=0 .
$$

According to Theorem 5.1, we can extract a sequence from $\left(\boldsymbol{u}_{n}\right)$ (still denoted $\left(\boldsymbol{u}_{n}\right)$ ) which converges to $\boldsymbol{u}$ in $\mathbf{L}^{2}(\Omega)$. By construction, we have $\|\boldsymbol{u}\|=1$. Then, we check easily that $\left(\boldsymbol{u}_{n}\right)$ converges to $\boldsymbol{u}$ in $\mathbf{H}_{N}(\operatorname{curl} ; \Omega)$ with $\operatorname{curl} \boldsymbol{u}=0$ a.e. in $\Omega$. Since $\partial \Omega$ is connected, one deduces (see [9], chapter 2, theorem 8) that there exists a scalar potential $\varphi \in \mathrm{H}_{0}^{1}(\Omega)$ such that $\boldsymbol{u}=\nabla \varphi$ in $\Omega$. Finally, we notice that $\operatorname{div}(\varepsilon \boldsymbol{u})=0$ and so $\operatorname{div}(\varepsilon \nabla \varphi)=0$. Lemma 3.1 implies $\varphi=0$ so $\boldsymbol{u}=0$. This leads to a contradiction because we must have $\|\boldsymbol{u}\|=1$.

\subsection{Compact embedding of $\mathbf{X}_{T}(\mu ; \Omega)$ in $\mathbf{L}^{2}(\Omega)$}

Now, let us work on the space of magnetic fields.

Theorem 5.3 Let $\Omega$ be a simply connected domain such that $\partial \Omega$ is connected. Assume $\left(\mathscr{H}_{\mu}\right)$ to be true. Then, the embedding of $\mathbf{X}_{T}(\mu ; \Omega)$ in $\mathbf{L}^{2}(\Omega)$ is compact.

Proof. Let $\left(\boldsymbol{u}_{n}\right)$ be a bounded sequence of elements of $\mathbf{X}_{T}(\mu ; \Omega)$. Define $f_{n}:=\operatorname{div}\left(\mu \boldsymbol{u}_{n}\right)$ and $\boldsymbol{F}_{n}:=\operatorname{curl} \boldsymbol{u}_{n}$. The sequence $\left(f_{n}\right)$ is bounded in $\mathrm{L}^{2}(\Omega)$ whereas $\left(\boldsymbol{F}_{n}\right)$ is bounded in $\mathbf{L}^{2}(\Omega)$. For all $n \in \mathbb{N}$, since $\int_{\Omega} \operatorname{div}\left(\mu \boldsymbol{u}_{n}\right)=\int_{\partial \Omega} \mu \boldsymbol{u}_{n} \cdot \boldsymbol{n}=0$, Lemma 3.5 allows us to build $\varphi_{n} \in \mathrm{H}_{\#}^{1}(\Omega)$ such that $\operatorname{div}\left(\mu \nabla \varphi_{n}\right)=\operatorname{div}\left(\mu \boldsymbol{u}_{n}\right)$. Observe next that $\mu\left(\boldsymbol{u}_{n}-\nabla \varphi_{n}\right)$ is a divergence free element of $\mathbf{L}^{2}(\Omega)$ such that $\mu\left(\boldsymbol{u}_{n}-\nabla \varphi_{n}\right) \cdot \boldsymbol{n}=0$ on $\partial \Omega$. Since $\Omega$ is simply connected, according to theorem 3.17 in [1], there exists a potential $\boldsymbol{w}_{n} \in \mathbf{V}_{N}(1 ; \Omega)$ such that $\operatorname{curl} \boldsymbol{w}_{n}=\mu\left(\boldsymbol{u}_{n}-\nabla \varphi_{n}\right)$. Thus, for all $n \in \mathbb{N}$, we have $\boldsymbol{u}_{n}=\nabla \varphi_{n}+\mu^{-1} \operatorname{curl} \boldsymbol{w}_{n}$

Then, we show as in the proof of Theorem 5.1, using the assumption $\left(\mathscr{H}_{\mu}\right)$, that we can extract subsequences from $\left(\nabla \varphi_{n}\right)$ and $\left(\operatorname{curl} \boldsymbol{w}_{n}\right)$ which converge in $\mathbf{L}^{2}(\Omega)$. 
As previously, this theorem allows us to obtain the

Corollary 5.4 Let $\Omega$ be a simply connected domain such that $\partial \Omega$ is connected. Assume $\left(\mathscr{H}_{\mu}\right)$ to be true. Then, there exists a constant $C$ such that

$$
\|\boldsymbol{u}\|^{2} \leq C\|\operatorname{curl} \boldsymbol{u}\|^{2}, \quad \forall \boldsymbol{u} \in \mathbf{V}_{T}(\mu ; \Omega) .
$$

Thus, the map $(\boldsymbol{u}, \boldsymbol{v}) \mapsto(\operatorname{curl} \boldsymbol{u}, \operatorname{curl} \boldsymbol{v})$ defines an inner product on $\mathbf{V}_{T}(\mu ; \Omega)$ and the associated norm is equivalent to the canonical norm $\boldsymbol{u} \mapsto(\boldsymbol{u}, \boldsymbol{u})_{\text {curl }}^{1 / 2}$.

\section{Well-posedness of Maxwell's equations}

We extend the results of Lemmas 4.1 and 4.5 to address the problem for the electric field (resp. magnetic field) when $\varepsilon$ (resp. $\mu$ ) changes sign. Thus, we precise Remark 4.4 (resp. Remark 4.8).

Lemma 6.1 Let $\Omega$ be a simply connected domain such that $\partial \Omega$ is connected. Assume $\left(\mathscr{H}_{\varepsilon}\right)$ and $\left(\mathscr{H}_{\mu}\right)$ to be true. Then:

- There exists an isomorphism $\mathbb{T}^{\varepsilon}$ of $\mathbf{V}_{N}(\varepsilon ; \Omega)$ such that, for all $\boldsymbol{u}, \boldsymbol{v} \in \mathbf{V}_{N}(\varepsilon ; \Omega)$,

$$
\left(\mu^{-1} \operatorname{curl} \boldsymbol{u}, \operatorname{curl} \mathbb{T}^{\varepsilon} \boldsymbol{v}\right)=\left(\mu^{-1} \operatorname{curl} \mathbb{T}^{\varepsilon} \boldsymbol{u}, \operatorname{curl} \boldsymbol{v}\right)=(\operatorname{curl} \boldsymbol{u}, \operatorname{curl} \boldsymbol{v}) .
$$

- There exists an isomorphism $\mathbb{T}^{\mu}$ of $\mathbf{V}_{T}(\mu ; \Omega)$ such that, for all $\boldsymbol{u}, \boldsymbol{v} \in \mathbf{V}_{T}(\mu ; \Omega)$,

$$
\left(\varepsilon^{-1} \operatorname{curl} \boldsymbol{u}, \operatorname{curl} \mathbb{T}^{\mu} \boldsymbol{v}\right)=\left(\varepsilon^{-1} \operatorname{curl} \mathbb{T}^{\mu} \boldsymbol{u}, \operatorname{curl} \boldsymbol{v}\right)=(\operatorname{curl} \boldsymbol{u}, \operatorname{curl} \boldsymbol{v}) .
$$

Proof. The first steps in the construction of the operators $\mathbb{T}^{\varepsilon}$ and $\mathbb{T}^{\mu}$ will be the same as those of Lemmas 4.1 and 4.5. However, we recall them for the sake of clarity. Of course, to obtain fields which belong to $\mathbf{V}_{N}(\varepsilon ; \Omega)$ and $\mathbf{V}_{T}(\mu ; \Omega)$ (instead of $\mathbf{V}_{N}(1 ; \Omega)$ and $\mathbf{V}_{T}(1 ; \Omega)$ ), we shall add one step.

$\star$ Definition of $\mathbb{T}^{\varepsilon}$. Consider $\boldsymbol{v} \in \mathbf{V}_{N}(\varepsilon ; \Omega)$.

i) Introduce $\varphi$ the unique element of $H_{\#}^{1}(\Omega)$ such that

$$
\int_{\Omega} \mu \nabla \varphi \cdot \nabla \varphi^{\prime}=\int_{\Omega} \mu \operatorname{curl} \boldsymbol{v} \cdot \nabla \varphi^{\prime}, \quad \forall \varphi^{\prime} \in \mathrm{H}_{\#}^{1}(\Omega) .
$$

The function $\varphi$ is well-defined since we have assumed $\left(\mathscr{H}_{\mu}\right)$ to be true.

ii) Remark next that $\mu(\operatorname{curl} \boldsymbol{v}-\nabla \varphi)$ is a divergence free element of $\mathbf{L}^{2}(\Omega)$ such that $\mu(\operatorname{curl} \boldsymbol{v}-\nabla \varphi) \cdot \boldsymbol{n}=0$ on $\partial \Omega$. Under the assumptions on the geometry, there exists a unique potential $\boldsymbol{\psi} \in \mathbf{V}_{N}(1 ; \Omega)$ such that $\operatorname{curl} \psi=\mu(\operatorname{curl} v-\nabla \varphi)$.

iii) Consider $\zeta$ the unique element of $\mathrm{H}_{0}^{1}(\Omega)$ such that

$$
\int_{\Omega} \varepsilon \nabla \zeta \cdot \nabla \zeta^{\prime}=\int_{\Omega} \varepsilon \boldsymbol{\psi} \cdot \nabla \zeta^{\prime}, \quad \forall \zeta^{\prime} \in \mathrm{H}_{0}^{1}(\Omega) .
$$

The function $\zeta$ is well-defined since we have assumed $\left(\mathscr{H}_{\varepsilon}\right)$ to be true.

iv) Finally, define the operator $\mathbb{T}^{\varepsilon}: \mathbf{V}_{N}(\varepsilon ; \Omega) \rightarrow \mathbf{V}_{N}(\varepsilon ; \Omega)$ such that $\mathbb{T}^{\varepsilon} \boldsymbol{v}=\boldsymbol{\psi}-\nabla \zeta$ for $\boldsymbol{v} \in \mathbf{V}_{N}(\varepsilon ; \Omega)$.

$\star$ Definition of $\mathbb{T}^{\mu}$. Consider $\boldsymbol{v} \in \mathbf{V}_{T}(\mu ; \Omega)$.

i) Introduce $\varphi$ the unique element of $\mathrm{H}_{0}^{1}(\Omega)$ such that

$$
\int_{\Omega} \varepsilon \nabla \varphi \cdot \nabla \varphi^{\prime}=\int_{\Omega} \varepsilon \operatorname{curl} \boldsymbol{v} \cdot \nabla \varphi^{\prime}, \quad \forall \varphi^{\prime} \in \mathrm{H}_{0}^{1}(\Omega) .
$$

The function $\varphi$ is well-defined since we have assumed $\left(\mathscr{H}_{\varepsilon}\right)$ to be true.

ii) Then, notice that $\varepsilon(\operatorname{curl} \boldsymbol{v}-\nabla \varphi)$ is a divergence free element of $\mathbf{L}^{2}(\Omega)$. Under the assumptions on the geometry, there exists a unique potential $\boldsymbol{\psi} \in \mathbf{V}_{T}(1 ; \Omega)$ such that $\operatorname{curl} \boldsymbol{\psi}=\varepsilon(\operatorname{curl} \boldsymbol{v}-\nabla \varphi)$.

iii) Consider $\zeta$ the unique element of $\mathrm{H}_{\#}^{1}(\Omega)$ such that

$$
\int_{\Omega} \mu \nabla \zeta \cdot \nabla \zeta^{\prime}=\int_{\Omega} \mu \psi \cdot \nabla \zeta^{\prime}, \quad \forall \zeta^{\prime} \in \mathrm{H}_{\#}^{1}(\Omega)
$$


The function $\zeta$ is well-defined since we have assumed $\left(\mathscr{H}_{\mu}\right)$ to be true.

iv) Finally, define the operator $\mathbb{T}^{\mu}: \mathbf{V}_{T}(\mu ; \Omega) \rightarrow \mathbf{V}_{T}(\mu ; \Omega)$ such that $\mathbb{T}^{\mu} \boldsymbol{v}=\boldsymbol{\psi}-\nabla \zeta$ for $\boldsymbol{v} \in \mathbf{V}_{T}(\mu ; \Omega)$.

A simple computation leads to (13) and (14). Proceeding as in Lemmas 4.1 and 4.5, and using Corollaries 5.2 and 5.4 which prove that $(\boldsymbol{u}, \boldsymbol{v}) \mapsto(\operatorname{curl} \boldsymbol{u}, \operatorname{curl} \boldsymbol{v})$ defines an inner product on $\mathbf{V}_{N}(\varepsilon ; \Omega)$ and on $\mathbf{V}_{T}(\mu ; \Omega)$, one shows a posteriori that $\mathbb{T}^{\varepsilon}$ and $\mathbb{T}^{\mu}$ are respectively isomorphisms of $\mathbf{V}_{N}(\varepsilon ; \Omega)$ and $\mathbf{V}_{T}(\mu ; \Omega)$.

We now have all the tools to prove the main result of this paper.

Theorem 6.2 Let $\Omega$ be a simply connected domain such that $\partial \Omega$ is connected. Assume also that

$$
\begin{gathered}
\left(\mathscr{H}_{\varepsilon}\right) \quad \begin{array}{c}
\text { There exists an isomorphism } T^{\varepsilon} \text { of } \mathrm{H}_{0}^{1}(\Omega) \text { such that } \\
\left|\left(\varepsilon \nabla \varphi, \nabla\left(T^{\varepsilon} \varphi\right)\right)\right| \geq C\|\varphi\|_{\mathrm{H}_{0}^{1}(\Omega)}^{2}, \quad \forall \varphi \in \mathrm{H}_{0}^{1}(\Omega) .
\end{array} \\
\left(\mathscr{H}_{\mu}\right) \quad \begin{array}{l}
\text { There exists an isomorphism } T^{\mu} \text { of } \mathrm{H}_{\#}^{1}(\Omega) \text { such that } \\
\left|\left(\mu \nabla \varphi, \nabla\left(T^{\mu} \varphi\right)\right)\right| \geq C\|\varphi\|_{\mathrm{H}_{\#}^{1}(\Omega)}^{2}, \quad \forall \varphi \in \mathrm{H}_{\#}^{1}(\Omega) .
\end{array}
\end{gathered}
$$

Then, the following results hold.

- There exists a unique solution to the problem for the electric field

$$
\mid \begin{aligned}
& \text { Find } \boldsymbol{E} \in \mathbf{V}_{N}(\varepsilon ; \Omega) \text { such that for all } \boldsymbol{E}^{\prime} \in \mathbf{V}_{N}(\varepsilon ; \Omega): \\
& \int_{\Omega} \mu^{-1} \operatorname{curl} \boldsymbol{E} \cdot \operatorname{curl} \boldsymbol{E}^{\prime}-\omega^{2} \varepsilon \boldsymbol{E} \cdot \boldsymbol{E}^{\prime}=i \omega \int_{\Omega} \boldsymbol{J} \cdot \boldsymbol{E}^{\prime},
\end{aligned}
$$

which continuously depends on the data $\boldsymbol{J}$, for all $\omega \in \mathbb{C} \backslash S$ where $S$ is a discrete set.

- There exists a unique solution to the problem for the magnetic field

$$
\begin{aligned}
& \text { Find } \boldsymbol{H} \in \mathbf{V}_{T}(\mu ; \Omega) \text { such that for all } \boldsymbol{H}^{\prime} \in \mathbf{V}_{T}(\mu ; \Omega) \text { : } \\
& \int_{\Omega} \varepsilon^{-1} \operatorname{curl} \boldsymbol{H} \cdot \operatorname{curl} \boldsymbol{H}^{\prime}-\omega^{2} \mu \boldsymbol{H} \cdot \boldsymbol{H}^{\prime}=\int_{\Omega} \varepsilon^{-1} \boldsymbol{J} \cdot \operatorname{curl} \boldsymbol{H}^{\prime}
\end{aligned}
$$

which continuously depends on the data $\boldsymbol{J}$, for all $\omega \in \mathbb{C} \backslash S$.

- Maxwell's equations (1)-(2) are uniquely solvable for all $\omega \in \mathbb{C}^{*} \backslash S$.

Proof. Let us begin with the first point. Lemma 6.1 ensures the existence of an isomorphism $\mathbb{T}^{\varepsilon}$ : $\mathbf{V}_{N}(\varepsilon ; \Omega) \rightarrow \mathbf{V}_{N}(\varepsilon ; \Omega)$ such that $(\boldsymbol{u}, \boldsymbol{v}) \mapsto\left(\mu^{-1} \operatorname{curl} \boldsymbol{u}, \operatorname{curl} \mathbb{T}^{\varepsilon} \boldsymbol{v}\right)$ is coercive on $\mathbf{V}_{N}(\varepsilon ; \Omega) \times \mathbf{V}_{N}(\varepsilon ; \Omega)$ Now, since $\mathbb{T}^{\varepsilon}$ is an isomorphism, $\boldsymbol{E}$ satisfies (15) if and only if $\boldsymbol{E}$ satisfies the problem

$$
\mid \begin{aligned}
& \text { Find } \boldsymbol{E} \in \mathbf{V}_{N}(\varepsilon ; \Omega) \text { such that for all } \boldsymbol{E}^{\prime} \in \mathbf{V}_{N}(\varepsilon ; \Omega) \text { : } \\
& \int_{\Omega} \mu^{-1} \operatorname{curl} \boldsymbol{E} \cdot \operatorname{curl}\left(\mathbb{T}^{\varepsilon} \boldsymbol{E}^{\prime}\right)-\omega^{2} \varepsilon \boldsymbol{E} \cdot\left(\mathbb{T}^{\varepsilon} \boldsymbol{E}^{\prime}\right)=i \omega \int_{\Omega} \boldsymbol{J} \cdot\left(\mathbb{T}^{\varepsilon} \boldsymbol{E}^{\prime}\right) .
\end{aligned}
$$

It only remains to observe that the Fredholm alternative holds for this problem because the embedding of $\mathbf{V}_{N}(\varepsilon ; \Omega)$ in $\mathbf{L}^{2}(\Omega)$ is compact by Theorem 5.1. The second point can be proved in the same way whereas the third statement can be obtained thanks to Theorems 3.3 and 3.7.

Remark 6.3 If in Eq. (1) one considers $\boldsymbol{J}$ such that $\operatorname{div} \boldsymbol{J} \neq 0$, it follows that $\operatorname{div}(\varepsilon \boldsymbol{E})=(i \omega)^{-1} \operatorname{div} \boldsymbol{J} \neq$ 0 . However, if one assumes that $\left(\mathscr{H}_{\varepsilon}\right)$ is true, one can solve (5) with right-hand side $f=(i \omega)^{-1} \operatorname{div} \boldsymbol{J}$. Then, one can proceed exactly as before with $(\boldsymbol{J}-i \omega \varepsilon \nabla \varphi, \boldsymbol{E}-\nabla \varphi)$ replacing $(\boldsymbol{J}, \boldsymbol{E})$ in Eq. (1).

\section{Illustrations}

We apply Theorem 6.2 in a few simple configurations. We focus on situations where the medium consists of two different materials. To model this problem, we assume that $\Omega$ is divided into two sub-domains $\Omega_{1}$ and $\Omega_{2}$ with $\bar{\Omega}=\overline{\Omega_{1}} \cup \overline{\Omega_{2}}$ et $\Omega_{1} \cap \Omega_{2}=\emptyset$. We denote $\Sigma:=\partial \Omega_{1} \backslash \partial \Omega=\partial \Omega_{2} \backslash \partial \Omega$. Let us introduce $\varepsilon_{1}$ and $\mu_{1}$ (resp. $\varepsilon_{2}$ and $\mu_{2}$ ) two elements of $L^{\infty}\left(\Omega_{1}\right)$ (resp. $L^{\infty}\left(\Omega_{2}\right)$ ). Define the functions $\varepsilon$ and $\mu$ such that $\left.\varepsilon\right|_{\Omega_{k}}=\varepsilon_{k}$ and $\left.\mu\right|_{\Omega_{k}}=\mu_{k}$ for $k=1,2$. We assume that $\Omega_{1}$ is filled with a positive material and that $\Omega_{2}$ is filled with a possibly negative material (for $\varepsilon$ and/or $\mu$ ). For that, we make the following assumptions: 

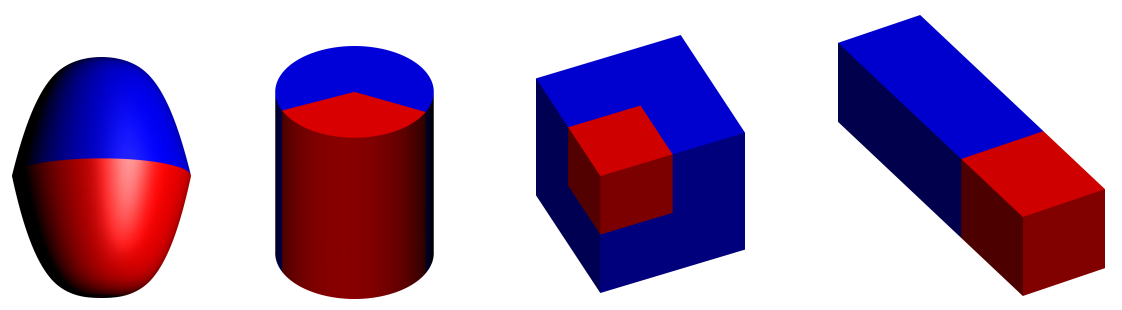

Figure 1: Canonical geometries: symmetric domain, prismatic edge, Fichera's corner, non symmetric cavity.

- there exists a constant $C$ s.t. $\varepsilon_{1} \geq C>0$ and $\mu_{1} \geq C>0$ a.e. in $\Omega_{1}$;

- there exists a constant $C$ s.t. $\varepsilon_{2} \geq C>0$ a.e. in $\Omega_{2}$ or $\varepsilon_{2} \leq-C<0$ a.e. in $\Omega_{2}$;

- there exists a constant $C$ s.t. $\mu_{2} \geq C>0$ a.e. in $\Omega_{2}$ or $\mu_{2} \leq-C<0$ a.e. in $\Omega_{2}$.

In particular, notice that $\varepsilon^{-1} \in \mathrm{L}^{\infty}(\Omega)$ and $\mu^{-1} \in \mathrm{L}^{\infty}(\Omega)$. Then, we define

$$
\sigma_{1}^{+}:=\sup _{\Omega_{1}} \sigma_{1}, \quad \sigma_{2}^{+}:=\sup _{\Omega_{2}}\left|\sigma_{2}\right|, \quad \sigma_{1}^{-}:=\inf _{\Omega_{1}} \sigma_{1} \quad \text { and } \quad \sigma_{2}^{-}:=\inf _{\Omega_{2}}\left|\sigma_{2}\right|, \quad \text { for } \sigma=\varepsilon, \mu .
$$

Generally speaking, if $v$ is a measurable function on $\Omega$, we use the notation $v_{k}:=\left.v\right|_{\Omega_{k}}, k=1,2$. For more details concerning the study of the scalar problems, we refer the reader to [2].

\subsection{Symmetric domain}

Let $\Omega$ be a symmetric domain, in the sense that $\Omega_{1}$ and $\Omega_{2}$ can be mapped from one to the other with the help of a reflection symmetry. Without loss of generality, we assume that the interface $\Sigma$ is included in the plane $z=0$ (see Figure 1, left, for an example). Consider the operators $R_{1}$ and $R_{2}$ respectively defined by $\left(R_{1} \varphi_{1}\right)(x, y, z)=\varphi_{1}(x, y,-z)$ and $\left(R_{2} \varphi_{2}\right)(x, y, z)=\varphi_{2}(x, y,-z)$ for $\varphi \in \mathrm{H}^{1}(\Omega)$. Define the operators $\mathrm{T}_{1}$ and $\mathrm{T}_{2}$ such that:

$$
\mathrm{T}_{1} \varphi=\left\{\begin{array}{ll}
\varphi_{1} & \text { in } \Omega_{1} \\
-\varphi_{2}+2 R_{1} \varphi_{1} & \text { in } \Omega_{2}
\end{array} ; \quad \mathrm{T}_{2} \varphi=\left\{\begin{array}{ll}
\varphi_{1}-2 R_{2} \varphi_{2} & \text { in } \Omega_{1} \\
-\varphi_{2} & \text { in } \Omega_{2}
\end{array} .\right.\right.
$$

By construction, $\mathrm{T}_{1} \varphi$ and $\mathrm{T}_{2} \varphi$ belong to $\mathrm{H}^{1}(\Omega)$. As $\mathrm{T}_{1} \circ \mathrm{T}_{1}=\mathrm{T}_{2} \circ \mathrm{T}_{2}=\mathrm{Id}$, we deduce that $\mathrm{T}_{1}$ and $\mathrm{T}_{2}$ are isomorphisms of $\mathrm{H}^{1}(\Omega)$. The restrictions $\mathrm{T}_{1}^{\varepsilon}$ and $\mathrm{T}_{2}^{\varepsilon}$ of $\mathrm{T}_{1}$ and $\mathrm{T}_{2}$ to $\mathrm{H}_{0}^{1}(\Omega)$ are isomorphisms of $\mathrm{H}_{0}^{1}(\Omega)$. Let us introduce the linear form $\gamma: \mathrm{H}^{1}(\Omega) \rightarrow \mathbb{R}$ such that $\gamma(\varphi)=\int_{\Omega} \varphi / \int_{\Omega} 1$. Note that $\operatorname{ker}(\gamma)=\mathrm{H}_{\#}^{1}(\Omega)$. Then, we define the operators $\mathrm{T}_{1}^{\mu}$ and $\mathrm{T}_{2}^{\mu}$ such that, for all $\varphi \in \mathrm{H}_{\#}^{1}(\Omega), \mathrm{T}_{1}^{\mu} \varphi=\mathrm{T}_{1} \varphi-\gamma\left(\mathrm{T}_{1} \varphi\right)$ and $\mathrm{T}_{2}^{\mu} \varphi=\mathrm{T}_{2} \varphi-\gamma\left(\mathrm{T}_{2} \varphi\right)$. Notice that $\mathrm{T}_{1}^{\mu} \varphi$ and $\mathrm{T}_{2}^{\mu} \varphi$ are elements of $\mathrm{H}_{\#}^{1}(\Omega)$. Moreover, we have

$$
\begin{aligned}
\mathrm{T}_{1}^{\mu}\left(\mathrm{T}_{1}^{\mu} \varphi\right)=\mathrm{T}_{1}^{\mu}\left(\mathrm{T}_{1} \varphi-\gamma\left(\mathrm{T}_{1} \varphi\right)\right) & =\mathrm{T}_{1}\left(\mathrm{~T}_{1} \varphi-\gamma\left(\mathrm{T}_{1} \varphi\right)\right)-\gamma\left(\mathrm{T}_{1}\left(\mathrm{~T}_{1} \varphi-\gamma\left(\mathrm{T}_{1} \varphi\right)\right)\right) \\
& =\varphi-\mathrm{T}_{1}\left(\gamma\left(\mathrm{T}_{1} \varphi\right)\right)-\gamma\left(\varphi-\mathrm{T}_{1}\left(\gamma\left(\mathrm{T}_{1} \varphi\right)\right)\right) \\
& =\varphi-\mathrm{T}_{1}\left(\gamma\left(\mathrm{T}_{1} \varphi\right)\right)+\gamma\left(\mathrm{T}_{1}\left(\gamma\left(\mathrm{T}_{1} \varphi\right)\right)\right)=\varphi .
\end{aligned}
$$

Thus, $\mathrm{T}_{1}^{\mu} \circ \mathrm{T}_{1}^{\mu}=\mathrm{Id}$. In the same way, we find $\mathrm{T}_{2}^{\mu} \circ \mathrm{T}_{2}^{\mu}=\mathrm{Id}$. Hence $\mathrm{T}_{1}^{\mu}$ and $\mathrm{T}_{2}^{\mu}$ are isomorphisms of $\mathrm{H}_{\#}^{1}(\Omega)$.

Proposition 7.1 (Symmetric domain)

Assume that $\varepsilon$ satisfies $\varepsilon \geq C>0$ a.e. in $\Omega$ or $\max \left(\varepsilon_{1}^{-} / \varepsilon_{2}^{+}, \varepsilon_{2}^{-} / \varepsilon_{1}^{+}\right)>1$.

Assume that $\mu$ satisfies $\mu \geq C>0$ a.e. in $\Omega$ or $\max \left(\mu_{1}^{-} / \mu_{2}^{+}, \mu_{2}^{-} / \mu_{1}^{+}\right)>1$.

Then, Maxwell's equations (1)-(2) are uniquely solvable for all $\omega \in \mathbb{C}^{*} \backslash S$ where $S$ is a discrete set.

Proof. Apply Theorem 6.2. To check that $\left(\mathscr{H}_{\varepsilon}\right)$ and $\left(\mathscr{H}_{\mu}\right)$ hold true, use the following table [2].

\begin{tabular}{|c|c|c|c|}
\hline For & $\varepsilon \geq C>0$ & $\varepsilon_{1}^{-} / \varepsilon_{2}^{+}>1$ & $\varepsilon_{2}^{-} / \varepsilon_{1}^{+}>1$ \\
\hline Take $^{\varepsilon}$ equal to & $\mathrm{Id}$ & $\mathrm{T}_{1}^{\varepsilon}$ & $\mathrm{T}_{2}^{\varepsilon}$ \\
\hline For & $\mu \geq C>0$ & $\mu_{1}^{-} / \mu_{2}^{+}>1$ & $\mu_{2}^{-} / \mu_{1}^{+}>1$ \\
\hline Take $\mathrm{T}^{\mu}$ equal to & $\mathrm{Id}$ & $\mathrm{T}_{1}^{\mu}$ & $\mathrm{T}_{2}^{\mu}$ \\
\hline
\end{tabular}


In the case where $\varepsilon$ and $\mu$ are constant on each side of the interface, the statement of Proposition 7.1 can be simplified.

Proposition 7.2 (Symmetric domain: PieceWise Constant Coefficients)

Assume that $\varepsilon_{1}, \varepsilon_{2}, \mu_{1}$ and $\mu_{2}$ are constant numbers. Then, if $\varepsilon_{2} / \varepsilon_{1}, \mu_{2} / \mu_{1} \in \mathbb{R}^{*} \backslash\{-1\}$, Maxwell's equations (1)-(2) are uniquely solvable for all $\omega \in \mathbb{C}^{*} \backslash S$ where $S$ is a discrete set.

\subsection{Prismatic edge}

Consider the geometry of Figure 1, middle-left. Introduce the cylindrical coordinates $(r, \theta, z)$ centered on the edge, so that the cartesian coordinates are mapped as $(x, y, z)=(r \cos \theta, r \sin \theta, z)$. Let $H>0$ denote the height of the cylinder, $R>0$ its radius. Given $0<\alpha<2 \pi$, define

$$
\begin{aligned}
& \Omega_{1}:=\{(r \cos \theta, r \sin \theta, z) \mid 0<r<R, 0<\theta<\alpha, 0<z<H\} ; \\
& \Omega_{2}:=\{(r \cos \theta, r \sin \theta, z) \mid 0<r<R, \alpha<\theta<2 \pi, 0<z<H\} .
\end{aligned}
$$

Introduce the two operators $R_{1}$ and $R_{2}$ such that $\left(R_{1} \varphi_{1}\right)(r, \theta, z)=\varphi_{1}\left(r, \frac{\alpha}{\alpha-2 \pi}(\theta-2 \pi), z\right)$ and $\left(R_{2} \varphi_{2}\right)(r, \theta, z)=$ $\varphi_{2}\left(r, \frac{\alpha-2 \pi}{\alpha} \theta+2 \pi, z\right)$ for $\varphi \in \mathrm{H}^{1}(\Omega)$.

Proceeding as for the case of the symmetric domain, one obtains the

Proposition 7.3 (PRismatic EDGe)

Define $I_{\alpha}:=\max \left(\frac{\alpha}{2 \pi-\alpha}, \frac{2 \pi-\alpha}{\alpha}\right)$.

Assume that $\varepsilon$ satisfies $\varepsilon \geq C>0$ a.e. in $\Omega$ or $\max \left(\varepsilon_{1}^{-} / \varepsilon_{2}^{+}, \varepsilon_{2}^{-} / \varepsilon_{1}^{+}\right)>I_{\alpha}$.

Assume that $\mu$ satisfies $\mu \geq C>0$ a.e. in $\Omega$ or $\max \left(\mu_{1}^{-} / \mu_{2}^{+}, \mu_{2}^{-} / \mu_{1}^{+}\right)>I_{\alpha}$.

Then, Maxwell's equations (1)-(2) are uniquely solvable for all $\omega \in \mathbb{C}^{*} \backslash S$ where $S$ is a discrete set.

Proposition 7.4 (Prismatic edge: Piecewise constant coefficients)

Assume that $\varepsilon_{1}, \varepsilon_{2}, \mu_{1}$ and $\mu_{2}$ are constant numbers. Define $I_{\alpha}:=\max \left(\frac{\alpha}{2 \pi-\alpha}, \frac{2 \pi-\alpha}{\alpha}\right)$. Then, if $\varepsilon_{2} / \varepsilon_{1}, \mu_{2} / \mu_{1} \in \mathbb{R}^{*} \backslash\left[-I_{\alpha} ;-1 / I_{\alpha}\right]$, Maxwell's equations (1)-(2) are uniquely solvable for all $\omega \in \mathbb{C}^{*} \backslash S$ where $S$ is a discrete set.

\subsection{Fichera corner}

Consider the geometry of Figure 1, middle-right. More precisely, define $\Omega:=(-1 ; 1)^{3}, \Omega_{1}:=(0 ; 1)^{3}$ and $\Omega_{2}:=\Omega \backslash \overline{\Omega_{1}}$.

Introduce the operator $R_{1}, R_{2}$, such that, for $\varphi \in \mathrm{H}^{1}(\Omega)$,

$$
\begin{aligned}
\left(R_{1} \varphi_{1}\right)(x, y, z)= & \begin{cases}\varphi_{1}(-x, y, z) & \text { in } \Omega_{2}^{1}:=(-1 ; 0) \times(0 ; 1)^{2} \\
\varphi_{1}(x,-y, z) & \text { in } \Omega_{2}^{2}:=(0 ; 1) \times(-1 ; 0) \times(0 ; 1) \\
\varphi_{1}(x, y,-z) & \text { in } \Omega_{2}^{3}:=(0 ; 1)^{2} \times(-1 ; 0) \\
\varphi_{1}(-x,-y, z) & \text { in } \Omega_{2}^{4}:=(-1 ; 0)^{2} \times(0 ; 1) \\
\varphi_{1}(-x, y,-z) & \text { in } \Omega_{2}^{5}:=(-1 ; 0) \times(0 ; 1) \times(-1 ; 0) \\
\varphi_{1}(x,-y,-z) & \text { in } \Omega_{2}^{6}:=(0 ; 1) \times(-1 ; 0)^{2} \\
\varphi_{1}(-x,-y,-z) & \text { in } \Omega_{2}^{7}:=(-1 ; 0)^{3}\end{cases} \\
\left(R_{2} \varphi_{2}\right)(x, y, z)= & \varphi_{2}^{1}(-x, y, z)+\varphi_{2}^{2}(x,-y, z)+\varphi_{2}^{3}(x, y,-z) \\
& -\varphi_{2}^{4}(-x,-y, z)-\varphi_{2}^{5}(-x, y,-z)-\varphi_{2}^{6}(x,-y,-z) \\
& +\varphi_{2}^{7}(-x,-y,-z) .
\end{aligned}
$$

Above, for $\ell=1 \ldots 7, \varphi_{2}^{\ell}$ is the restriction of $\varphi_{2}$ to $\Omega_{2}^{\ell}$.

Again, proceeding as for the case of the symmetric domain, one obtains the

Proposition 7.5 (FICHERA's CORNER)

Assume that $\varepsilon$ satisfies $\varepsilon \geq C>0$ a.e. in $\Omega$ or $\max \left(\varepsilon_{1}^{-} / \varepsilon_{2}^{+}, \varepsilon_{2}^{-} / \varepsilon_{1}^{+}\right)>7$.

Assume that $\mu$ satisfies $\mu \geq C>0$ a.e. in $\Omega$ or $\max \left(\mu_{1}^{-} / \mu_{2}^{+}, \mu_{2}^{-} / \mu_{1}^{+}\right)>7$.

Then, Maxwell's equations (1)-(2) are uniquely solvable for all $\omega \in \mathbb{C}^{*} \backslash S$ where $S$ is a discrete set.

Proposition 7.6 (FicherA's CORNER: PIECEWISE CONSTANT COEFFICIENTS)

Assume that $\varepsilon_{1}, \varepsilon_{2}, \mu_{1}$ and $\mu_{2}$ are constant numbers. Then, if $\varepsilon_{2} / \varepsilon_{1}, \mu_{2} / \mu_{1} \in \mathbb{R}^{*} \backslash[-7 ;-1 / 7]$, Maxwell's equations (1)-(2) are uniquely solvable for all $\omega \in \mathbb{C}^{*} \backslash S$ where $S$ is a discrete set. 


\subsection{Non symmetric cavity}

Let us consider the non symmetric cavity of Figure 1. More precisely, define $\Omega:=\{(x, y, z) \in(-a ; b) \times$ $(0 ; 1) \times(0 ; 1)\}, \Omega_{1}:=(-a ; 0) \times(0 ; 1) \times(0 ; 1)$ and $\Omega_{2}:=(0 ; b) \times(0 ; 1) \times(0 ; 1)$ with $a>0$ and $b>0$. The interface $\Sigma$ is then equal to $\{0\} \times(0 ; 1) \times(0 ; 1)$. Assume that $\varepsilon_{1}, \varepsilon_{2}, \mu_{1}$ and $\mu_{2}$ are constant numbers.

Recall that in $\S 3.1$, we have defined the operator $A^{\varepsilon}$ such that, for all $\varphi, \varphi^{\prime} \in \mathrm{H}_{0}^{1}(\Omega),\left(\nabla\left(A^{\varepsilon} \varphi\right), \nabla \varphi^{\prime}\right)=$ $\left(\varepsilon \nabla \varphi, \nabla \varphi^{\prime}\right)$ whereas in $\S 3.2$, we have introduced the operator $A^{\mu}$ such that, for all $\varphi, \varphi^{\prime} \in \mathrm{H}_{\#}^{1}(\Omega)$, $\left(\nabla\left(A^{\mu} \varphi\right), \nabla \varphi^{\prime}\right)=\left(\mu \nabla \varphi, \nabla \varphi^{\prime}\right)$. For this particular geometry, we know (see [2]) that the operator $A^{\varepsilon}$ (resp. $A^{\mu}$ ) is Fredholm of index 0 (see Definition 8.1 below) if and only if $\varepsilon_{2} / \varepsilon_{1} \neq-1$ (resp. $\mu_{2} / \mu_{1} \neq-1$ ). To apply Theorem 6.2 , we need $A^{\varepsilon}$ and $A^{\mu}$ to be isomorphisms. Therefore, we study here the question of the injectivity of $A^{\varepsilon}$ and $A^{\mu}$. Let us start with $A^{\varepsilon}$. Consider $\varphi$ an element of $\mathrm{H}_{0}^{1}(\Omega)$ such that $A^{\varepsilon} \varphi=0$. The pair $\left(\varphi_{1}, \varphi_{2}\right)$ satisfies the equations

$$
\begin{array}{llllll}
\Delta \varphi_{1}=0 & \text { in } \Omega_{1} ; & \varphi_{1}-\varphi_{2} & = & 0 & \text { on } \Sigma \\
\Delta \varphi_{2}=0 & \text { in } \Omega_{2} ; & \varepsilon_{1} \partial_{x} \varphi_{1}-\varepsilon_{2} \partial_{x} \varphi_{2} & =0 & \text { on } \Sigma .
\end{array}
$$

Decomposing $\varphi_{1}$ and $\varphi_{2}$ in Fourier series (the family $\{(y, z) \mapsto \sin (m \pi y) \sin (n \pi z)\}_{m, n=1}^{\infty}$ is a basis of $\left.L^{2}((0 ; 1) \times(0 ; 1))\right)$, one obtains

$$
\begin{aligned}
\varphi_{1}(x, y, z) & =\sum_{n=1}^{\infty} \sum_{m=1}^{\infty} \varphi_{1}^{m n} \sinh \left(\sqrt{m^{2}+n^{2}} \pi(x+a)\right) \sin (m \pi y) \sin (n \pi z) \\
\text { and } \quad \varphi_{2}(x, y, z) & =\sum_{n=1}^{\infty} \sum_{m=1}^{\infty} \varphi_{2}^{m n} \sinh \left(\sqrt{m^{2}+n^{2}} \pi(x-b)\right) \sin (m \pi y) \sin (n \pi z),
\end{aligned}
$$

where $\varphi_{1}^{m n}$ and $\varphi_{2}^{m n}$ are constant numbers. Besides, the transmission conditions imply,

$$
\forall(m, n) \in \mathbb{N}^{*} \times \mathbb{N}^{*}, \mid \begin{array}{ll}
\varphi_{1}^{m n} \sinh \left(\sqrt{m^{2}+n^{2}} \pi a\right)=-\varphi_{2}^{m n} \sinh \left(\sqrt{m^{2}+n^{2}} \pi b\right) \\
\varphi_{1}^{m n} \varepsilon_{1} \cosh \left(\sqrt{m^{2}+n^{2}} \pi a\right)=\varphi_{2}^{m n} \varepsilon_{2} \cosh \left(\sqrt{m^{2}+n^{2}} \pi b\right)
\end{array} .
$$

For each $(m, n) \in \mathbb{N}^{*} \times \mathbb{N}^{*}$, there exists a non trivial solution to the system (16) (in $\left.\left(\varphi_{1}^{m n}, \varphi_{2}^{m n}\right)\right)$ if and only if

$$
\begin{aligned}
0 & =\varepsilon_{2} \sinh \left(\sqrt{m^{2}+n^{2}} \pi a\right) \cosh \left(\sqrt{m^{2}+n^{2}} \pi b\right)+\varepsilon_{1} \sinh \left(\sqrt{m^{2}+n^{2}} \pi b\right) \cosh \left(\sqrt{m^{2}+n^{2}} \pi a\right) \\
\Leftrightarrow \quad \varepsilon_{2} / \varepsilon_{1} & =-\frac{\tanh \left(\sqrt{m^{2}+n^{2}} \pi b\right)}{\tanh \left(\sqrt{m^{2}+n^{2}} \pi a\right)} .
\end{aligned}
$$

Consequently, $A^{\varepsilon}: \mathrm{H}_{0}^{1}(\Omega) \rightarrow \mathrm{H}_{0}^{1}(\Omega)$ is an isomorphism if and only if $\varepsilon_{2} / \varepsilon_{1}$ is not an element of

$$
\mathscr{S}_{\varepsilon}:=\left\{-\tanh \left(\sqrt{m^{2}+n^{2}} \pi b\right) / \tanh \left(\sqrt{m^{2}+n^{2}} \pi a\right),(m, n) \in \mathbb{N}^{*} \times \mathbb{N}^{*}\right\} \cup\{-1\} .
$$

Following the same way, exchanging the "sin" by "cos" and the "sinh" by "cosh" to satisfy the Neumann condition, we prove that $A^{\mu}: \mathrm{H}_{\#}^{1}(\Omega) \rightarrow \mathrm{H}_{\#}^{1}(\Omega)$ is an isomorphism if and only if $\mu_{2} / \mu_{1}$ is not an element of

$$
\mathscr{S}_{\mu}:=\left\{-\tanh \left(\sqrt{m^{2}+n^{2}} \pi a\right) / \tanh \left(\sqrt{m^{2}+n^{2}} \pi b\right),(m, n) \in \mathbb{N}^{*} \times \mathbb{N}^{*}\right\} \cup\{-1\} .
$$

Remark 7.7 The map $g: z \mapsto-\tanh (z \pi b) / \tanh (z \pi a)$ is continuous, strictly decreasing if $a>b$ and strictly increasing if $a<b$. Besides, we have $\lim _{z \rightarrow+\infty} g(z)=-1$. As a consequence, -1 is an accumulation point of both sets $\mathscr{S}_{\varepsilon}$ and $\mathscr{S}_{\mu}$.

Remark 7.8 For this particular 3D geometry, we obtain a result specific to 2D configurations (see [3]): the problem with Dirichlet boundary condition for the coefficient $\varepsilon$ is well-posed if and only if the problem with Neumann boundary condition is well-posed for the coefficient $\mu:=\varepsilon^{-1}$.

We deduce the

Proposition 7.9 (Non symmetric CaVity: Piecewise Constant Coefficients) Assume that $\varepsilon_{1}, \varepsilon_{2}, \mu_{1}$ and $\mu_{2}$ are constant numbers. Assume that $\varepsilon_{2} / \varepsilon_{1} \in \mathbb{R}^{*} \backslash \mathscr{S}_{\varepsilon}$ and $\mu_{2} / \mu_{1} \in \mathbb{R}^{*} \backslash \mathscr{S}_{\mu}$, with $\mathscr{S}_{\varepsilon}$ and $\mathscr{S}_{\mu}$ respectively defined in (17) and (18). Then, Maxwell's equations (1)-(2) are uniquely solvable for all $\omega \in \mathbb{C}^{*} \backslash S$ where $S$ is a discrete set. 


\section{Relaxing the main hypotheses}

To prove the previous results, we relied extensively on two types of assumptions. On the one hand, hypotheses $\left(\mathscr{H}_{\varepsilon}\right)$ and $\left(\mathscr{H}_{\mu}\right)$ ensure that the scalar problems are well-posed. On the other hand, the domain $\Omega$ is supposed to be simply connected, with a connected boundary. We would like now to relax these assumptions.

Concerning the hypotheses on the geometry, the difficulty is well-known (see for instance [9]). For instance, if the boundary $\partial \Omega$ is not connected, the space $\mathbf{V}_{N}(\varepsilon ; \Omega)$ contains non-trivial curl free fields $\nabla \varphi$, so that $\boldsymbol{u} \mapsto(\boldsymbol{u}, \boldsymbol{u})_{\text {curl }}^{1 / 2}$ is not a norm on $\mathbf{V}_{N}(\varepsilon ; \Omega)$ anymore. The same occurs for $\mathbf{V}_{T}(\mu ; \Omega)$ when $\Omega$ is not simply connected.

At first glance, relaxing hypotheses $\left(\mathscr{H}_{\varepsilon}\right)$ and $\left(\mathscr{H}_{\mu}\right)$ has similar consequences. For instance, suppose that the homogeneous scalar problem (5) with $f=0$ has a non-trivial solution $\tilde{\varphi}$ : the non-trivial curl free field $\nabla \tilde{\varphi}$ belongs to $\mathbf{V}_{N}(\varepsilon ; \Omega)$. Once more, $\boldsymbol{u} \mapsto(\boldsymbol{u}, \boldsymbol{u})_{\text {curl }}^{1 / 2}$ is not a norm on $\mathbf{V}_{N}(\varepsilon ; \Omega)$.

However, we observe a fundamental difference between the scalar potentials which are built in the two cases: $\varphi \notin \mathrm{H}_{0}^{1}(\Omega)$, whereas $\tilde{\varphi} \in \mathrm{H}_{0}^{1}(\Omega)$. As a consequence, the field $\tilde{\boldsymbol{E}}=\nabla \tilde{\varphi}$ is such that:

$$
\int_{\Omega} \varepsilon \tilde{\boldsymbol{E}} \cdot \boldsymbol{E}^{\prime}=0, \quad \forall \boldsymbol{E}^{\prime} \in \mathbf{V}_{N}(\varepsilon ; \Omega)
$$

which is not the case for $\boldsymbol{E}=\nabla \varphi$. So, $\tilde{\boldsymbol{E}}$ is a solution to the homogeneous problem $(\boldsymbol{J}=0)$ for the electric field (6) stated in $\mathbf{V}_{N}(\varepsilon ; \Omega)$ but not to the homogeneous problem (3) stated in $\mathbf{H}_{N}(\mathbf{c u r l} ; \Omega)$. In other words, when the scalar problems have non-trivial kernels, Theorems 3.3 and 3.7 (equivalence with the original Maxwell's problem) are no longer true.

Summing up, we see that the difficulties which occur when relaxing either hypotheses on the geometry or hypotheses on the scalar problem present some similarities (existence of admissible fields which are both divergence free and curl free) but also some fundamental differences (formulations in $\mathbf{V}_{N}(\varepsilon ; \Omega$ ) and $\mathbf{V}_{T}(\mu ; \Omega)$ are no longer equivalent to the original problem when the scalar problem have kernels). Since the non-injectivity of the scalar problems is a difficulty which is specific to the presence of signchanging coefficients $\varepsilon$ and/or $\mu$, it will be treated first in Subsection 8.1. Then, in Subsection 8.2, we shall check that the usual treatment for non-trivial geometries can be extended to sign-changing coefficients.

\subsection{Extension to non injective scalar problems}

\subsubsection{Non injective scalar problems: a new functional framework}

We have introduced the sesquilinear forms $a^{\varepsilon}$ and $a^{\mu}$ such that

$$
\begin{array}{ll}
a^{\varepsilon}\left(\varphi, \varphi^{\prime}\right)=\left(\varepsilon \nabla \varphi, \nabla \varphi^{\prime}\right), & \forall \varphi, \varphi^{\prime} \in \mathrm{H}_{0}^{1}(\Omega) ; \\
a^{\mu}\left(\varphi, \varphi^{\prime}\right)=\left(\mu \nabla \varphi, \nabla \varphi^{\prime}\right), & \forall \varphi, \varphi^{\prime} \in \mathrm{H}_{\#}^{1}(\Omega) .
\end{array}
$$

With the Riesz representation theorem, we have defined the operators

$$
\begin{array}{lll}
A^{\varepsilon}: \mathrm{H}_{0}^{1}(\Omega) \rightarrow \mathrm{H}_{0}^{1}(\Omega) & \text { s.t. }\left(\nabla\left(A^{\varepsilon} \varphi\right), \nabla \varphi^{\prime}\right)=a^{\varepsilon}\left(\varphi, \varphi^{\prime}\right), \quad \forall \varphi, \varphi^{\prime} \in \mathrm{H}_{0}^{1}(\Omega) ; \\
\text { and } \quad A^{\mu}: \mathrm{H}_{\#}^{1}(\Omega) \rightarrow \mathrm{H}_{\#}^{1}(\Omega) & \text { s.t. }\left(\nabla\left(A^{\mu} \varphi\right), \nabla \varphi^{\prime}\right)=a^{\mu}\left(\varphi, \varphi^{\prime}\right), \quad \forall \varphi, \varphi^{\prime} \in \mathrm{H}_{\#}^{1}(\Omega) .
\end{array}
$$

Theorem 6.2 indicates that Maxwell's equations (1)-(2) are well-posed in the Fredholm sense when $A^{\varepsilon}$ and $A^{\mu}$ are isomorphisms. In this section, we want to study the case when $A^{\varepsilon}$ and $A^{\mu}$ are non injective Fredholm operators of index 0 . Let us recall the definition of a Fredholm operator (see [34, 22]).

Definition 8.1 Let $\mathrm{X}$ and $\mathrm{Y}$ be two Banach spaces, and let $L: \mathrm{X} \rightarrow \mathrm{Y}$ be a continuous linear map. The operator $L$ is said to be a Fredholm operator if and only if the following two conditions are fulfilled

i) $\operatorname{dim}(\operatorname{ker} L)<\infty$ and $\operatorname{range} L$ is closed;

ii) $\operatorname{dim}(\operatorname{coker} L)<\infty$ where coker $L:=(\mathrm{Y} / \operatorname{range} L)$. 
Besides, the index of a Fredholm operator $L$ is defined by $\operatorname{ind} L=\operatorname{dim}(\operatorname{ker} L)-\operatorname{dim}(\operatorname{coker} L)$.

For the example of the non symmetric cavity described in $\S 7.4$, this corresponds to consider the situation where $\kappa_{\varepsilon} \in \mathscr{S}_{\varepsilon} \backslash\{-1\}$ and $\kappa_{\mu} \in \mathscr{S}_{\mu} \backslash\{-1\}$. For the sake of brevity, we will focus on configurations where $A^{\varepsilon}$ and $A^{\mu}$ both have a kernel non reduced to zero. When only one of these two operators is not injective, the study of the Maxwell's equations can be easily inferred from the one we present.

Up to the end of this section, we assume that the physical parameters $\varepsilon, \mu$ and the geometry are such that $A^{\varepsilon}: \mathrm{H}_{0}^{1}(\Omega) \rightarrow \mathrm{H}_{0}^{1}(\Omega)$ and $A^{\mu}: \mathrm{H}_{\#}^{1}(\Omega) \rightarrow \mathrm{H}_{\#}^{1}(\Omega)$ are non injective Fredholm operators of index 0 . Let us introduce $\left\{\lambda_{i}^{\varepsilon}\right\}_{i=1}^{N^{\varepsilon}}$ a basis of ker $A^{\varepsilon}$ such that $\left(\nabla \lambda_{i}^{\varepsilon}, \nabla \lambda_{j}^{\varepsilon}\right)=\delta_{i j}$ and $\left\{\lambda_{i}^{\mu}\right\}_{i=1}^{N^{\mu}}$ a basis of ker $A^{\mu}$ such that $\left(\nabla \lambda_{i}^{\mu}, \nabla \lambda_{j}^{\mu}\right)=\delta_{i j}$. Define the spaces $\mathrm{S}^{\varepsilon}$ and $\mathrm{S}^{\mu}$ such that

$$
\mathrm{H}_{0}^{1}(\Omega)=\operatorname{ker} A^{\varepsilon} \stackrel{\perp}{\oplus} \mathrm{S}^{\varepsilon} \quad \text { and } \quad \mathrm{H}_{\#}^{1}(\Omega)=\operatorname{ker} A^{\mu} \stackrel{\perp}{\oplus} \mathrm{S}^{\mu} .
$$

Consider the sesquilinear forms $\tilde{a}^{\varepsilon}$ and $\tilde{a}^{\mu}$ such that

$$
\begin{aligned}
& \tilde{a}^{\varepsilon}\left(\varphi, \varphi^{\prime}\right)=\left(\varepsilon \nabla \varphi, \nabla \varphi^{\prime}\right), \quad \forall \varphi, \varphi^{\prime} \in \mathrm{S}^{\varepsilon} ; \\
& \tilde{a}^{\mu}\left(\varphi, \varphi^{\prime}\right)=\left(\mu \nabla \varphi, \nabla \varphi^{\prime}\right), \quad \forall \varphi, \varphi^{\prime} \in \mathrm{S}^{\mu} .
\end{aligned}
$$

With the Riesz representation theorem, define the operators

$$
\begin{array}{llll}
\tilde{A}^{\varepsilon}: \mathrm{S}^{\varepsilon} \rightarrow \mathrm{S}^{\varepsilon} & \text { s.t. }\left(\nabla\left(\tilde{A}^{\varepsilon} \varphi\right), \nabla \varphi^{\prime}\right)=\tilde{a}^{\varepsilon}\left(\varphi, \varphi^{\prime}\right), & \forall \varphi, \varphi^{\prime} \in \mathrm{S}^{\varepsilon} \\
\text { and } & \tilde{A}^{\mu}: \mathrm{S}^{\mu} \rightarrow \mathrm{S}^{\mu} & \text { s.t. }\left(\nabla\left(\tilde{A}^{\mu} \varphi\right), \nabla \varphi^{\prime}\right)=\tilde{a}^{\mu}\left(\varphi, \varphi^{\prime}\right), & \forall \varphi, \varphi^{\prime} \in \mathrm{S}^{\mu} .
\end{array}
$$

Classically (see [22]), one has the

Proposition 8.2 The operators $\tilde{A}^{\varepsilon}: \mathrm{S}^{\varepsilon} \rightarrow \mathrm{S}^{\varepsilon}$ and $\tilde{A}^{\mu}: \mathrm{S}^{\mu} \rightarrow \mathrm{S}^{\mu}$ are isomorphisms.

As mentioned in the introduction of Section 8, Theorems 3.3 and 3.7 do not hold anymore. Indeed, $\operatorname{span}\left(\nabla \lambda_{1}^{\varepsilon}, \ldots, \nabla \lambda_{N^{\varepsilon}}^{\varepsilon}\right)$ is included in the kernel of problem (6), stated in $\mathbf{V}_{N}(\varepsilon ; \Omega)$, but not in the kernel of the original problem (3), stated in $\mathbf{H}_{N}(\mathbf{c u r l} ; \Omega)$. Our objective is therefore to write variational formulations of Maxwell's problems in some spaces different from $\mathbf{V}_{N}(\varepsilon ; \Omega)$ and $\mathbf{V}_{T}(\mu ; \Omega)$ in order to eliminate these artificial kernels. A way to achieve that is to enrich the usual spaces by setting

$$
\begin{aligned}
\tilde{\mathbf{V}}_{N}(\varepsilon ; \Omega) & :=\left\{\boldsymbol{u} \in \mathbf{H}_{N}(\operatorname{curl} ; \Omega) \mid(\varepsilon \boldsymbol{u}, \nabla \varphi)=0, \forall \varphi \in S^{\varepsilon}\right\} \\
\tilde{\mathbf{V}}_{T}(\mu ; \Omega) & :=\left\{\boldsymbol{u} \in \mathbf{H}(\operatorname{curl} ; \Omega) \mid(\mu \boldsymbol{u}, \nabla \varphi)=0, \forall \varphi \in S^{\mu}\right\} .
\end{aligned}
$$

Notice that we have $\mathbf{V}_{N}(\varepsilon ; \Omega) \subset \tilde{\mathbf{V}}_{N}(\varepsilon ; \Omega)$ and $\mathbf{V}_{T}(\mu ; \Omega) \subset \tilde{\mathbf{V}}_{T}(\mu ; \Omega)$. Let us precise the relation between these spaces.

Lemma 8.3 For $i=1 \ldots N^{\varepsilon}$, there exists $\boldsymbol{\Lambda}_{i}^{\varepsilon} \in \tilde{\mathbf{V}}_{N}(\varepsilon ; \Omega)$ such that $\left(\varepsilon \boldsymbol{\Lambda}_{i}^{\varepsilon}, \nabla \lambda_{j}^{\varepsilon}\right)=\delta_{i j}$, for $j=1 \ldots N^{\varepsilon}$. We deduce

$$
\tilde{\mathbf{V}}_{N}(\varepsilon ; \Omega)=\mathbf{V}_{N}(\varepsilon ; \Omega) \oplus \operatorname{span}\left(\boldsymbol{\Lambda}_{i}^{\varepsilon}\right)_{i=1}^{N^{\varepsilon}} .
$$

Proof. For $j=1 \ldots N^{\varepsilon}$, let us introduce the linear form $\ell_{j}$ on $\tilde{\mathbf{V}}_{N}(\varepsilon ; \Omega)$ defined by $\ell_{j}(\boldsymbol{v})=\left(\varepsilon \boldsymbol{v}, \nabla \lambda_{j}^{\varepsilon}\right)$. Let us prove that the family $\ell_{1}, \ldots, \ell_{N^{\varepsilon}}$ is free. Let $\alpha_{1}, \ldots, \alpha_{N^{\varepsilon}}$ be $N^{\varepsilon}$ constants such that $\sum_{j=1}^{N^{\varepsilon}} \overline{\alpha_{j}} \ell_{j}=0$. For all $\boldsymbol{v} \in \tilde{\mathbf{V}}_{N}(\varepsilon ; \Omega)$, we have $\left(\varepsilon \boldsymbol{v}, \sum_{j=1}^{N^{\varepsilon}} \alpha_{j} \nabla \lambda_{j}^{\varepsilon}\right)=0$. This implies $\left(\varepsilon \boldsymbol{w}, \sum_{j=1}^{N^{\varepsilon}} \alpha_{j} \nabla \lambda_{j}^{\varepsilon}\right)=0$ for all $\boldsymbol{w} \in \mathbf{H}_{N}(\operatorname{curl} ; \Omega)$. Indeed, for $\boldsymbol{w} \in \mathbf{H}_{N}(\mathbf{c u r l} ; \Omega)$, let us introduce $\varphi \in \mathrm{S}^{\varepsilon}$ the function such that $\left(\varepsilon \nabla \varphi, \nabla \varphi^{\prime}\right)=\left(\varepsilon \boldsymbol{w}, \nabla \varphi^{\prime}\right)$ for all $\varphi^{\prime} \in \mathrm{S}^{\varepsilon} ; \varphi$ is well-defined according to Proposition 8.2. We have $\boldsymbol{w}-\nabla \varphi \in \tilde{\mathbf{V}}_{N}(\varepsilon ; \Omega)$. Since $\left(\varepsilon \nabla \varphi, \nabla \lambda_{j}^{\varepsilon}\right)=0, j=1 \ldots N^{\varepsilon}$, we obtain $\left(\varepsilon \boldsymbol{w}, \sum_{j=1}^{N^{\varepsilon}} \alpha_{j} \nabla \lambda_{j}^{\varepsilon}\right)=0$.

Since $\mathscr{C}_{0}^{\infty}(\Omega)^{2} \subset \mathbf{H}_{N}(\mathbf{c u r l} ; \Omega)$ is dense in $\mathbf{L}^{2}(\Omega)$, we deduce $\sum_{j=1}^{N^{\varepsilon}} \overline{\alpha_{j}} \nabla \lambda_{j}^{\varepsilon}=0$. The family $\lambda_{1}^{\varepsilon}, \ldots, \lambda_{N^{\varepsilon}}^{\varepsilon}$ constitutes a basis of ker $A^{\varepsilon}$, so we have $\alpha_{1}=\cdots=\alpha_{N^{\varepsilon}}=0: \ell_{1}, \ldots, \ell_{N^{\varepsilon}}$ is free. Then Theorem 8.4 (see [20, lemma 4.12]) hereafter ensures the existence of the $\left(\boldsymbol{\Lambda}_{i}^{\varepsilon}\right)$. Finally, for all $\boldsymbol{v} \in \tilde{\mathbf{V}}_{N}(\varepsilon ; \Omega)$, $\boldsymbol{v}-\sum_{j=1}^{N^{\varepsilon}} \ell_{j}(\boldsymbol{v}) \boldsymbol{\Lambda}_{j}^{\varepsilon}$ belongs to $\mathbf{V}_{N}(\varepsilon ; \Omega)$, which ends the proof.

Theorem 8.4 Let $\mathrm{V}$ be a normed vector space. For all free family $\ell^{1}, \ldots, \ell^{n} \in \mathrm{V}^{*}$, there exist $x^{1}, \ldots, x^{n} \in$ $\mathrm{V}$ such that

$$
\ell^{i}\left(x^{j}\right)=\delta^{i j} \quad \text { with } \delta^{i j}=1 \text { if } i=j \text { and } \delta^{i j}=0 \text { if } i \neq j .
$$


Proceeding in the same way, one can prove the

Lemma 8.5 For $i=1 \ldots N^{\mu}$, there exists $\boldsymbol{\Lambda}_{i}^{\mu} \in \tilde{\mathbf{V}}_{T}(\mu ; \Omega)$ such that $\left(\mu \boldsymbol{\Lambda}_{i}^{\mu}, \nabla \lambda_{j}^{\mu}\right)=\delta_{i j}$, for $j=1 \ldots N^{\mu}$. We deduce

$$
\tilde{\mathbf{V}}_{T}(\mu ; \Omega)=\mathbf{V}_{T}(\mu ; \Omega) \oplus \operatorname{span}\left(\boldsymbol{\Lambda}_{i}^{\mu}\right)_{i=1}^{N^{\mu}} .
$$

\subsubsection{Non injective scalar problems: equivalent formulations}

Now, we can give equivalent formulations to problem $(1)-(2)$ in the spaces $\tilde{\mathbf{V}}_{N}(\varepsilon ; \Omega)$ and $\tilde{\mathbf{V}}_{T}(\mu ; \Omega)$.

Theorem 8.6 Assume that $\omega \neq 0$ and that the operator $A^{\varepsilon}: \mathrm{H}_{0}^{1}(\Omega) \rightarrow \mathrm{H}_{0}^{1}(\Omega)$ is Fredholm of index 0 with a non trivial kernel $\operatorname{span}\left\{\lambda_{i}^{\varepsilon}\right\}_{i=1}^{N^{\varepsilon}}$. Let $\tilde{\mathbf{V}}_{N}(\varepsilon ; \Omega)$ be defined as in (19).

1) If $(\boldsymbol{E}, \boldsymbol{H})$ satisfies (1)-(2) then $\boldsymbol{E}$ is a solution of the problem

$$
\mid \begin{aligned}
& \text { Find } \boldsymbol{E} \in \tilde{\mathbf{V}}_{N}(\varepsilon ; \Omega) \quad \text { such that for all } \boldsymbol{E}^{\prime} \in \tilde{\mathbf{V}}_{N}(\varepsilon ; \Omega): \\
& \int_{\Omega} \mu^{-1} \operatorname{curl} \boldsymbol{E} \cdot \operatorname{curl} \boldsymbol{E}^{\prime}-\omega^{2} \varepsilon \boldsymbol{E} \cdot \boldsymbol{E}^{\prime}=i \omega \int_{\Omega} \boldsymbol{J} \cdot \boldsymbol{E}^{\prime}
\end{aligned}
$$

2) If $\boldsymbol{E}$ satisfies (20) then $\left(\boldsymbol{E},(i \omega \mu)^{-1} \operatorname{curl} \boldsymbol{E}\right)$ satisfies (1)-(2).

Proof. Let us focus on the proof 2$) \Rightarrow 1$ ). For that, let us show that if $\boldsymbol{E} \in \tilde{\mathbf{V}}_{N}(\varepsilon ; \Omega) \subset \mathbf{H}_{N}(\operatorname{curl} ; \Omega)$ satisfies (20) then $\boldsymbol{E}$ is a solution to the problem (7) set in $\mathbf{H}_{N}(\mathbf{c u r l} ; \Omega)$. For all $\boldsymbol{E}^{\prime}$ in $\mathbf{H}_{N}(\mathbf{c u r l} ; \Omega)$, Proposition 8.2 indicates that we can build $\varphi \in \mathrm{S}^{\varepsilon}$ such that $\tilde{a}^{\varepsilon}\left(\varphi, \varphi^{\prime}\right)=\left(\varepsilon \boldsymbol{E}^{\prime}, \nabla \varphi^{\prime}\right)$, for all $\varphi^{\prime} \in \mathrm{S}^{\varepsilon}$. The element $\boldsymbol{E}^{\prime}-\nabla \varphi$ belongs to $\tilde{\mathbf{V}}_{N}(\varepsilon ; \Omega)$. Observing that $(\varepsilon \boldsymbol{E}, \nabla \varphi)=0$ and $(\boldsymbol{J}, \nabla \varphi)=0$ (recall that $\operatorname{div} \boldsymbol{J}=0$ ), one obtains

$$
\int_{\Omega} \mu^{-1} \operatorname{curl} \boldsymbol{E} \cdot \operatorname{curl} \boldsymbol{E}^{\prime}-\omega^{2} \varepsilon \boldsymbol{E} \cdot \boldsymbol{E}^{\prime}=i \omega \int_{\Omega} \boldsymbol{J} \cdot \boldsymbol{E}^{\prime} .
$$

Therefore, if $\boldsymbol{E}$ satisfies (20) then $\left(\boldsymbol{E},(i \omega \mu)^{-1} \operatorname{curl} \boldsymbol{E}\right)$ satisfies (1)-(2).

Adapting the proof of Theorem 8.6, one obtains the

Theorem 8.7 Assume that $\omega \neq 0$ and that the operator $A^{\mu}: \mathrm{H}_{\#}^{1}(\Omega) \rightarrow \mathrm{H}_{\#}^{1}(\Omega)$ is Fredholm of index 0 with a non trivial kernel $\operatorname{span}\left\{\lambda_{i}^{\mu}\right\}_{i=1}^{N^{\mu}}$. Let $\tilde{\mathbf{V}}_{T}(\mu ; \Omega)$ be defined as in (19).

1) If $(\boldsymbol{E}, \boldsymbol{H})$ satisfies (1)-(2) then $\boldsymbol{H}$ is a solution of the problem

$$
\begin{aligned}
& \text { Find } \boldsymbol{H} \in \tilde{\mathbf{V}}_{T}(\mu ; \Omega) \text { such that for all } \boldsymbol{H}^{\prime} \in \tilde{\mathbf{V}}_{T}(\mu ; \Omega) \text { : } \\
& \int_{\Omega} \varepsilon^{-1} \operatorname{curl} \boldsymbol{H} \cdot \operatorname{curl} \boldsymbol{H}^{\prime}-\omega^{2} \mu \boldsymbol{H} \cdot \boldsymbol{H}^{\prime}=\int_{\Omega} \varepsilon^{-1} \boldsymbol{J} \cdot \operatorname{curl} \boldsymbol{H}^{\prime}
\end{aligned}
$$

2) If $\boldsymbol{H}$ satisfies (21) then $\left(i(\omega \varepsilon)^{-1}(\operatorname{curl} \boldsymbol{H}-\boldsymbol{J}), \boldsymbol{H}\right)$ satisfies (1)-(2).

Now, we wish to study formulations (20) and (21). For that, we need some new compactness results.

\subsubsection{Non injective scalar problems: compactness results}

Let us start by proving the compactness result needed to study the problem for the electric field.

Theorem 8.8 Let $\Omega$ be a simply connected domain such that $\partial \Omega$ is connected. Then the embedding of $\tilde{\mathbf{V}}_{N}(\varepsilon ; \Omega)$ in $\mathbf{L}^{2}(\Omega)$ is compact.

Proof. Let $\left(\boldsymbol{u}_{n}\right)$ be a bounded sequence of $\tilde{\mathbf{V}}_{N}(\varepsilon ; \Omega)$. For all $n \in \mathbb{N}$, using Lemma 8.3, define $\boldsymbol{v}_{n} \in \mathbf{V}_{N}(\varepsilon ; \Omega)$ and $\left(\alpha_{i n}\right) \in \mathbb{C}^{N^{\varepsilon}}\left(\alpha_{i n}=\ell_{i}\left(\boldsymbol{u}_{n}\right)\right)$ such that $\boldsymbol{u}_{n}=\boldsymbol{v}_{n}+\sum_{i=1}^{N^{\varepsilon}} \alpha_{i n} \boldsymbol{\Lambda}_{i}^{\varepsilon}$. To prove Theorem 8.8, it is sufficient to show that we can extract a subsequence from $\left(\boldsymbol{v}_{n}\right)$ which converges in $\mathbf{L}^{2}(\Omega)$. Define $\boldsymbol{F}_{n}=\operatorname{curl} \boldsymbol{v}_{n}$. The sequence $\left(\boldsymbol{F}_{n}\right)$ is bounded in $\mathbf{L}^{2}(\Omega)$. For all $n \in \mathbb{N}$, we have $\operatorname{div}\left(\varepsilon \boldsymbol{v}_{n}\right)=0$. Since $\partial \Omega$ is connected, there exists (see [1], theorem 3.12) $\boldsymbol{w}_{n} \in \mathbf{V}_{T}(1 ; \Omega)$ such that curl $\boldsymbol{w}_{n}=\varepsilon \boldsymbol{v}_{n}$. Thus, for all $n \in \mathbb{N}$, we can write $\boldsymbol{v}_{n}=\varepsilon^{-1} \operatorname{curl} \boldsymbol{w}_{n}$. Let us show we can extract a sequence from $\left(\operatorname{curl} \boldsymbol{w}_{n}\right)$ which converges in $\mathbf{L}^{2}(\Omega)$.

We know that $\boldsymbol{w} \mapsto\|\operatorname{curl} \boldsymbol{w}\|$ defines a norm on $\mathbf{V}_{T}(1 ; \Omega)$. Consequently, the sequence $\left(\boldsymbol{w}_{n}\right)$ is 
bounded in $\mathbf{V}_{T}(1 ; \Omega)$. Since $\operatorname{curl} \varepsilon^{-1} \operatorname{curl} \boldsymbol{w}_{n}=\boldsymbol{F}_{n}$ in $\Omega$ and $\left(\varepsilon^{-1} \operatorname{curl} \boldsymbol{w}_{n}\right) \times \boldsymbol{n}=0$ on $\partial \Omega$, one has $\left(\varepsilon^{-1} \operatorname{curl} \boldsymbol{w}_{n}, \operatorname{curl} \boldsymbol{w}^{\prime}\right)=\left(\boldsymbol{F}_{n}, \boldsymbol{w}^{\prime}\right)$, for all $\boldsymbol{w}^{\prime} \in \mathbf{V}_{T}(1 ; \Omega)$.

Now, let us build a continuous operator $\mathbb{T}$ from $\mathbf{V}_{T}(1 ; \Omega)$ to itself, to restore some property of positivity. Consider $\boldsymbol{w} \in \mathbf{V}_{T}(1 ; \Omega)$.

i) First, define $\varphi$ the unique element of $S^{\varepsilon}$ such that

$$
\int_{\Omega} \varepsilon \nabla \varphi \cdot \nabla \varphi^{\prime}=\int_{\Omega} \varepsilon\left(\operatorname{curl} \boldsymbol{w}-\sum_{i=1}^{N^{\varepsilon}} \beta_{i} \boldsymbol{\Lambda}_{i}^{\varepsilon}\right) \cdot \nabla \varphi^{\prime}, \quad \forall \varphi^{\prime} \in \mathrm{S}^{\varepsilon}
$$

where $\beta_{i}=\left(\varepsilon \operatorname{curl} \boldsymbol{w}, \nabla \lambda_{i}^{\varepsilon}\right)$. The function $\varphi$ is well-defined since $\tilde{A}^{\varepsilon}: \mathrm{S}^{\varepsilon} \rightarrow \mathrm{S}^{\varepsilon}$ is an isomorphism.

ii) Thanks to the definition of the coefficients $\left(\beta_{i}\right)$, notice that $\varepsilon\left(\operatorname{curl} \boldsymbol{w}-\sum_{i=1}^{N^{\varepsilon}} \beta_{i} \boldsymbol{\Lambda}_{i}^{\varepsilon}-\nabla \varphi\right)$ is a divergence free element of $\mathbf{L}^{2}(\Omega)$. Since $\Omega$ is simply connected and since $\partial \Omega$ is connected, according to theorem 3.12 in [1], there exists a unique potential $\mathbb{T} \boldsymbol{w} \in \mathbf{V}_{T}(1 ; \Omega)$ such that $\operatorname{curl} \mathbb{T} \boldsymbol{w}=\varepsilon\left(\operatorname{curl} \boldsymbol{w}-\sum_{i=1}^{N^{\varepsilon}} \beta_{i} \boldsymbol{\Lambda}_{i}^{\varepsilon}-\nabla \varphi\right)$. This defines a bounded operator $\mathbb{T}: \mathbf{V}_{T}(1 ; \Omega) \rightarrow \mathbf{V}_{T}(1 ; \Omega)$.

Since $\mathbb{T}$ is continuous, the sequence $\left(\mathbb{T} \boldsymbol{w}_{n}\right)$ is bounded in $\mathbf{V}_{T}(1 ; \Omega)$. So, we can extract a subsequence from $\left(\boldsymbol{w}_{n}\right)$, still denoted $\left(\boldsymbol{w}_{n}\right)$, such that $\left(\mathbb{T} \boldsymbol{w}_{n}\right)$ converges in $\mathbf{L}^{2}(\Omega)$. Since for $i=1 \ldots N^{\varepsilon}$, the sequence $\left(\beta_{\text {in }}\right)$, with $\beta_{\text {in }}=\left(\varepsilon \operatorname{curl} \boldsymbol{w}_{n}, \nabla \lambda_{i}^{\varepsilon}\right)$, is bounded in $\mathbb{C}$, we can extract a subsequence from $\left(\boldsymbol{w}_{n}\right)$, still denoted $\left(\boldsymbol{w}_{n}\right)$, such that $\left(\beta_{i n}\right)$ converges. Introducing $\boldsymbol{w}_{n m}:=\boldsymbol{w}_{n}-\boldsymbol{w}_{m}, \boldsymbol{F}_{n m}:=\boldsymbol{F}_{n}-\boldsymbol{F}_{m}$ and $\beta_{\text {inm }}:=\beta_{\text {in }}-\beta_{\text {im }}$, one finds

$$
\begin{aligned}
\left|\left(\boldsymbol{F}_{n m}, \mathbb{T} \boldsymbol{w}_{n m}\right)\right| & =\left|\left(\varepsilon^{-1} \operatorname{curl} \boldsymbol{w}_{n m}, \operatorname{curl} \mathbb{T} \boldsymbol{w}_{n m}\right)\right| \\
& \geq\left\|\operatorname{curl} \boldsymbol{w}_{n m}\right\|^{2}-\sum_{i=1}^{N^{\varepsilon}}\left|\beta_{i n m}\right|\left|\left(\operatorname{curl} \boldsymbol{w}_{n m}, \boldsymbol{\Lambda}_{i}^{\varepsilon}\right)\right|
\end{aligned}
$$

This estimate proves that ( $\left.\operatorname{curl} \boldsymbol{w}_{n}\right)$ is a Cauchy sequence in $\mathbf{L}^{2}(\Omega)$, so it converges. Thus, we can extract a subsequence from $\left(\boldsymbol{v}_{n}\right)=\left(\varepsilon^{-1} \operatorname{curl} \boldsymbol{w}_{n}\right)$ which converges in $\mathbf{L}^{2}(\Omega)$.

Proceeding in the same way, we can prove the

Theorem 8.9 Let $\Omega$ be a simply connected domain such that $\partial \Omega$ is connected. Then the embedding of $\tilde{\mathbf{V}}_{T}(\mu ; \Omega)$ in $\mathbf{L}^{2}(\Omega)$ is compact.

\subsubsection{Non injective scalar problems: well-posedness of Maxwell's equations}

With the Riesz representation theorem, let us introduce the bounded operators $\tilde{\mathscr{A}}_{N}(\omega): \tilde{\mathbf{V}}_{N}(\varepsilon ; \Omega) \rightarrow$ $\tilde{\mathbf{V}}_{N}(\varepsilon ; \Omega)$ and $\tilde{\mathscr{A}}_{T}(\omega): \tilde{\mathbf{V}}_{T}(\mu ; \Omega) \rightarrow \tilde{\mathbf{V}}_{T}(\mu ; \Omega)$ such that for all $\boldsymbol{E}, \boldsymbol{E}^{\prime} \in \tilde{\mathbf{V}}_{N}(\varepsilon ; \Omega)$ and for all $\boldsymbol{H}, \boldsymbol{H}^{\prime} \in$ $\tilde{\mathbf{V}}_{T}(\mu ; \Omega)$,

$$
\begin{aligned}
& \left(\tilde{\mathscr{A}}_{N}(\omega) \boldsymbol{E}, \boldsymbol{E}^{\prime}\right)_{\text {curl }}=\left(\mu^{-1} \operatorname{curl} \boldsymbol{E}, \operatorname{curl} \boldsymbol{E}^{\prime}\right)-\omega^{2}\left(\varepsilon \boldsymbol{E}, \boldsymbol{E}^{\prime}\right), \\
& \left(\tilde{\mathscr{A}}_{T}(\omega) \boldsymbol{H}, \boldsymbol{H}^{\prime}\right)_{\text {curl }}=\left(\varepsilon^{-1} \operatorname{curl} \boldsymbol{H}, \operatorname{curl} \boldsymbol{H}^{\prime}\right)-\omega^{2}\left(\mu \boldsymbol{H}, \boldsymbol{H}^{\prime}\right) \text {. }
\end{aligned}
$$

Now, let us state the main result when the geometry and the physical coefficients $\varepsilon, \mu$ are such that the scalar problems are well-posed in the Fredholm sense with a non trivial kernel.

Theorem 8.10 Let $\Omega$ be a simply connected domain such that $\partial \Omega$ is connected. Consider $\boldsymbol{J} \in \mathbf{L}^{2}(\Omega)$ such that $\operatorname{div} \boldsymbol{J}=0$.

Assume that the operator $A^{\varepsilon}: \mathrm{H}_{0}^{1}(\Omega) \rightarrow \mathrm{H}_{0}^{1}(\Omega)$ is Fredholm of index 0 with a non trivial kernel $\operatorname{span}\left\{\lambda_{i}^{\varepsilon}\right\}_{i=1}^{N^{\varepsilon}}$.

Assume that the operator $A^{\mu}: \mathrm{H}_{\#}^{1}(\Omega) \rightarrow \mathrm{H}_{\#}^{1}(\Omega)$ is Fredholm of index 0 with a non trivial kernel $\operatorname{span}\left\{\lambda_{i}^{\mu}\right\}_{i=1}^{N^{\mu}}$.

Then, the following results hold.

- For all $\omega \in \mathbb{C}$, the operator $\tilde{\mathscr{A}}_{N}(\omega): \tilde{\mathbf{V}}_{N}(\varepsilon ; \Omega) \rightarrow \tilde{\mathbf{V}}_{N}(\varepsilon ; \Omega)$ is a Fredholm operator of index 0 . Moreover, for $\omega \in \mathbb{C}^{*}, \boldsymbol{E} \in \tilde{\mathbf{V}}_{N}(\varepsilon ; \Omega)$ satisfies $\left(\tilde{\mathscr{A}}_{N}(\omega) \boldsymbol{E}, \boldsymbol{E}^{\prime}\right)_{\text {curl }}=i \omega\left(\boldsymbol{J}, \boldsymbol{E}^{\prime}\right)$, for all $\boldsymbol{E}^{\prime} \in \tilde{\mathbf{V}}_{N}(\varepsilon ; \Omega)$, if and only if the pair $\left(\boldsymbol{E},(i \omega \mu)^{-1} \mathbf{c u r l} \boldsymbol{E}\right)$ satisfies the Maxwell's equations (1)-(2).

- For all $\omega \in \mathbb{C}, \tilde{\mathscr{A}}_{T}(\omega): \tilde{\mathbf{V}}_{T}(\mu ; \Omega) \rightarrow \tilde{\mathbf{V}}_{T}(\mu ; \Omega)$ is a Fredholm operator of index 0. Moreover, for $\omega \in \mathbb{C}^{*}, \boldsymbol{H} \in \tilde{\mathbf{V}}_{T}(\mu ; \Omega)$ satisfies $\left(\tilde{\mathscr{A}}_{T}(\omega) \boldsymbol{H}, \boldsymbol{H}^{\prime}\right)_{\mathbf{c u r l}}=\left(\varepsilon^{-1} \boldsymbol{J}, \mathbf{c u r l} \boldsymbol{H}^{\prime}\right)$, for all $\boldsymbol{H}^{\prime} \in \tilde{\mathbf{V}}_{T}(\mu ; \Omega)$, if and only if the pair $\left(i(\omega \varepsilon)^{-1}(\operatorname{curl} \boldsymbol{H}-\boldsymbol{J}), \boldsymbol{H}\right)$ satisfies the Maxwell's equations (1)-(2). 
Proof. Let us prove that $\tilde{\mathscr{A}}_{N}(\omega)$ is a Fredholm operator of index 0 . For all $\omega \in \mathbb{C}$, using Theorem 8.8, we can prove that $\tilde{\mathscr{A}}_{N}(\omega)-\mathscr{\mathscr { A }}_{N}(0)$ is a compact operator of $\tilde{\mathbf{V}}_{N}(\varepsilon ; \Omega)$. Consequently, according to [22, theorem 2.26], $\tilde{\mathscr{A}}_{N}(\omega)$ is a Fredholm operator of index 0 if and only if $\tilde{\mathscr{A}}_{N}(0)$ is a Fredholm operator of index 0 . In the sequel, we work on $\tilde{\mathscr{A}}_{N}(0)$. We build a bounded operator $\tilde{\mathbb{T}}^{\varepsilon}: \tilde{\mathbf{V}}_{N}(\varepsilon ; \Omega) \rightarrow \tilde{\mathbf{V}}_{N}(\varepsilon ; \Omega)$ to restore some positivity up to a compact perturbation. Let us consider $\boldsymbol{u} \in \tilde{\mathbf{V}}_{N}(\varepsilon ; \Omega)$.

i) First, define $\varphi$ the unique element of $\mathrm{S}^{\mu}$ such that

$$
\int_{\Omega} \mu \nabla \varphi \cdot \nabla \varphi^{\prime}=\int_{\Omega} \mu\left(\operatorname{curl} \boldsymbol{u}-\sum_{i=1}^{N^{\mu}} \beta_{i} \boldsymbol{\Lambda}_{i}^{\mu}\right) \cdot \nabla \varphi^{\prime}, \quad \forall \varphi^{\prime} \in \mathrm{S}^{\mu},
$$

where $\beta_{i}=\left(\mu \operatorname{curl} \boldsymbol{u}, \nabla \lambda_{i}^{\mu}\right)$. The function $\varphi$ is well-defined since $\tilde{A}^{\mu}: \mathrm{S}^{\mu} \rightarrow \mathrm{S}^{\mu}$ is an isomorphism.

ii) Then, notice that $\mu\left(\operatorname{curl} \boldsymbol{u}-\sum_{i=1}^{N^{\mu}} \beta_{i} \boldsymbol{\Lambda}_{i}^{\mu}-\nabla \varphi\right)$ is a divergence free element of $\mathbf{L}^{2}(\Omega)$ such that $\mu\left(\operatorname{curl} \boldsymbol{u}-\sum_{i=1}^{N^{\mu}} \beta_{i} \boldsymbol{\Lambda}_{i}^{\mu}-\nabla \varphi\right) \cdot \boldsymbol{n}=0$ on $\partial \Omega$. Since $\Omega$ is simply connected and since $\partial \Omega$ is connected, according to theorem 3.17 in [1], there exists a unique potential $\psi \in \mathbf{V}_{N}(1 ; \Omega)$ such that $\operatorname{curl} \psi=$ $\mu\left(\operatorname{curl} \boldsymbol{u}-\sum_{i=1}^{N^{\mu}} \beta_{i} \boldsymbol{\Lambda}_{i}^{\mu}-\nabla \varphi\right)$.

iii) Consider $\zeta$ the unique element of $S^{\varepsilon}$ such that

$$
\int_{\Omega} \varepsilon \nabla \zeta \cdot \nabla \zeta^{\prime}=\int_{\Omega} \varepsilon \psi \cdot \nabla \zeta^{\prime}, \quad \forall \zeta^{\prime} \in \mathrm{S}^{\varepsilon} .
$$

The function $\zeta$ is well-defined since $\tilde{A}^{\varepsilon}: \mathrm{S}^{\varepsilon} \rightarrow \mathrm{S}^{\varepsilon}$ is an isomorphism.

iv) Finally, define the operator $\tilde{\mathbb{T}}^{\varepsilon}: \tilde{\mathbf{V}}_{N}(\varepsilon ; \Omega) \rightarrow \tilde{\mathbf{V}}_{N}(\varepsilon ; \Omega)$ such that $\tilde{\mathbb{T}}^{\varepsilon} \boldsymbol{u}=\boldsymbol{\psi}-\nabla \zeta$ and the operator $\tilde{K}^{\varepsilon}: \tilde{\mathbf{V}}_{N}(\varepsilon ; \Omega) \rightarrow \tilde{\mathbf{V}}_{N}(\varepsilon ; \Omega)$ such that

$$
\left(\tilde{K}^{\varepsilon} \boldsymbol{u}, \boldsymbol{v}\right)_{\operatorname{curl}}=(\boldsymbol{u}, \boldsymbol{v})+\sum_{i=1}^{N^{\mu}}\left(\mu \operatorname{curl} \boldsymbol{u}, \nabla \lambda_{i}^{\mu}\right)\left(\boldsymbol{\Lambda}_{i}^{\mu}, \operatorname{curl} \boldsymbol{v}\right), \quad \forall \boldsymbol{v} \in \tilde{\mathbf{V}}_{N}(\varepsilon ; \Omega) .
$$

According to Theorem 8.8, we know that the embedding of $\tilde{\mathbf{V}}_{N}(\varepsilon ; \Omega)$ in $\mathbf{L}^{2}(\Omega)$ is compact. Consequently, $\tilde{K}^{\varepsilon}$ is the sum of a compact operator and a finite rank operator. Therefore, it is a compact operator. Now, for all $\boldsymbol{u}, \boldsymbol{v} \in \tilde{\mathbf{V}}_{N}(\varepsilon ; \Omega)$, we obtain

$$
\begin{aligned}
\left(\tilde{\mathscr{A}}_{N}(0)\left(\tilde{\mathbb{T}}^{\varepsilon} \boldsymbol{u}\right), \boldsymbol{v}\right)_{\text {curl }} & =\left(\mu^{-1} \operatorname{curl}\left(\tilde{\mathbb{T}}^{\varepsilon} \boldsymbol{u}\right), \operatorname{curl} \boldsymbol{v}\right) \\
& =(\operatorname{curl} \boldsymbol{u}, \operatorname{curl} \boldsymbol{v})+(\boldsymbol{u}, \boldsymbol{v})-\left(\tilde{K}^{\varepsilon} \boldsymbol{u}, \boldsymbol{v}\right)_{\text {curl }} .
\end{aligned}
$$

We deduce $\tilde{\mathscr{A}}_{T}(0) \circ \tilde{\mathbb{T}}^{\varepsilon}+\tilde{K}^{\varepsilon}=\mathrm{Id}$. This proves that $\tilde{\mathbb{T}}^{\varepsilon}$ is a right parametrix for $\tilde{\mathscr{A}}_{N}(0)$. Thus, the selfadjoint operator $\tilde{\mathscr{A}}_{N}(0)$ is Fredholm of index 0 (use [22, Lemma 2.23]). In the same way, we prove that $\tilde{\mathscr{A}}_{T}(\omega): \tilde{\mathbf{V}}_{T}(\mu ; \Omega) \rightarrow \tilde{\mathbf{V}}_{T}(\mu ; \Omega)$ is a Fredholm operator of index 0 for all $\omega \in \mathbb{C}$. Finally, the equivalence with Maxwell's equations (1)-(2) comes from Theorems 8.6 and 8.7.

Remark 8.11 To apply the analytic Fredholm theorem to prove that Maxwell's equations (1)-(2) are uniquely solvable for all $\omega \in \mathbb{C}^{*} \backslash S$ where $S$ is a discrete set, it remains to show that there exists $\omega \in \mathbb{C}$ such that $\tilde{\mathscr{A}}_{N}(\omega)$ or $\tilde{\mathscr{A}}_{T}(\omega)$ is invertible. However, we have not been able to prove this result.

\subsection{Extension to a non trivial geometry}

\subsubsection{Non trivial geometry: a decomposition of the functional spaces}

Classical configurations for Maxwell's equations include non-topologically trivial domains, and/or domains with a non-connected boundary. We study these configurations here. To avoid multiple sub-cases, we focus our work on the case of a non simply connected domain whose boundary is not connected. Figure 2 presents an example of such a geometry. In these geometries, the functions $\boldsymbol{u}$ of $\mathbf{V}_{N}(\varepsilon ; \Omega)$ (resp. $\mathbf{V}_{T}(\mu ; \Omega)$ ) do not necessarily write under the form $\boldsymbol{u}=\varepsilon^{-1} \operatorname{curl} \boldsymbol{\psi}$ (resp. $\boldsymbol{u}=\mu^{-1} \operatorname{curl} \boldsymbol{\psi}$ ) where $\boldsymbol{\psi}$ belongs to $\mathbf{V}_{T}(1 ; \Omega)$ (resp. $\mathbf{V}_{N}(1 ; \Omega)$ ). However, imposing more restrictive conditions to the functions of $\mathbf{V}_{N}(\varepsilon ; \Omega)$ and $\mathbf{V}_{T}(\mu ; \Omega)$, we can recover these results of existence for the potentials.

To introduce the spaces adapted to the study of Maxwell's equations in this kind of domains, we use the notations of [1]. 


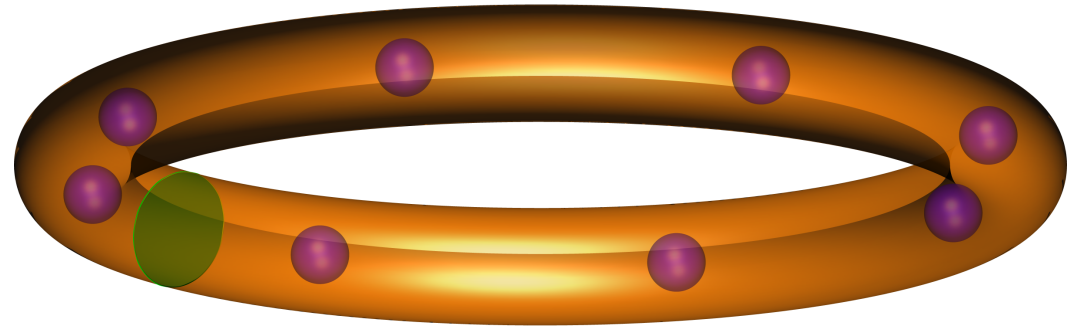

Figure 2: An example of domain which is not simply connected and whose boundary is not connected. The domain is made of the torus without the purple inclusions. It is not simply connected because of the toroidal structure. The boundary is not connected because the boundary of the torus and the ones of the spheres are not connected. The green disk represents a cut $\Sigma_{1}$ which is such that $\Omega \backslash \Sigma_{1}$ is simply connected.

Notations for domains with a non connected boundary. We denote $\Gamma_{i}, i=0 \ldots I$, the connected components of the boundary $\partial \Omega$. Since we assume that $\partial \Omega$ is not connected, we have $I \geq 1$. Let us introduce

$$
\mathrm{H}_{\Gamma}^{1}(\Omega):=\left\{\varphi \in \mathrm{H}^{1}(\Omega) \mid \varphi_{\mid \Gamma_{0}}=0, \varphi_{\mid \Gamma_{i}}=c s t, i=1 \ldots I\right\} .
$$

Let us start by characterizing this space. Using a lifting function, we prove the

Proposition 8.12 Assume $\left(\mathscr{H}_{\varepsilon}\right)$ to be true. Then, for $i=1 \ldots I$, there exists a unique solution $p_{i}$ to the problem

Find $p_{i} \in \mathrm{H}_{\Gamma}^{1}(\Omega)$ such that: $\operatorname{div}\left(\varepsilon \nabla p_{i}\right)=0 \quad$ in $\Omega$

$p_{i}=\delta_{i k} \quad$ on $\Gamma_{k}, k=1 \ldots I$.

Then, we have

$$
\mathrm{H}_{\Gamma}^{1}(\Omega)=\mathrm{H}_{0}^{1}(\Omega) \oplus \operatorname{span}\left(p_{i}\right)_{i=1}^{I}
$$

Define

$$
\hat{\mathbf{V}}_{N}(\varepsilon ; \Omega):=\left\{\boldsymbol{u} \in \mathbf{H}_{N}(\operatorname{curl} ; \Omega) \mid(\varepsilon \boldsymbol{u}, \nabla \varphi)=0, \forall \varphi \in \mathrm{H}_{\Gamma}^{1}(\Omega)\right\} .
$$

Notice that $\hat{\mathbf{V}}_{N}(\varepsilon ; \Omega) \subset \mathbf{V}_{N}(\varepsilon ; \Omega)$. Let us precise the link between these two spaces.

Lemma 8.13 Assume $\left(\mathscr{H}_{\varepsilon}\right)$ to be true. For $i=1 \ldots I$, there exists $\boldsymbol{P}_{i} \in \mathbf{V}_{N}(\varepsilon ; \Omega)$ such that $\left(\varepsilon \boldsymbol{P}_{i}, \nabla p_{k}\right)=$ $\delta_{i k}$, for $k=1 \ldots I$. We deduce

$$
\begin{aligned}
\mathbf{V}_{N}(\varepsilon ; \Omega) & =\hat{\mathbf{V}}_{N}(\varepsilon ; \Omega) \oplus \operatorname{span}\left(\boldsymbol{P}_{i}\right)_{i=1}^{I} \\
\text { and } \quad \mathbf{H}_{N}(\operatorname{curl} ; \Omega) & =\hat{\mathbf{V}}_{N}(\varepsilon ; \Omega) \oplus \operatorname{span}\left(\boldsymbol{P}_{i}\right)_{i=1}^{I} \oplus \nabla \mathrm{H}_{0}^{1}(\Omega) .
\end{aligned}
$$

Proof. For $k=1 \ldots I$, let us define the linear form $\ell_{k}$ on $\mathbf{V}_{N}(\varepsilon ; \Omega)$ such that $\ell_{k}(\boldsymbol{v})=\left(\varepsilon \boldsymbol{v}, \nabla p_{k}\right)$. Let us prove that the family $\ell_{1}, \ldots, \ell_{I}$ is free. Consider $I$ constants $\alpha_{1}, \ldots, \alpha_{I}$ such that $\sum_{k=1}^{I} \overline{\alpha_{k}} \ell_{k}=0$. For all $\boldsymbol{v} \in \mathbf{V}_{N}(\varepsilon ; \Omega)$, we have $\left(\varepsilon \boldsymbol{v}, \sum_{k=1}^{I} \alpha_{k} \nabla p_{k}\right)=0$. We deduce $\left(\varepsilon \boldsymbol{w}, \sum_{k=1}^{I} \alpha_{k} \nabla p_{k}\right)=0$ for all $\boldsymbol{w} \in \mathbf{H}_{N}(\operatorname{curl} ; \Omega)$. Indeed, if $\boldsymbol{w} \in \mathbf{H}_{N}(\operatorname{curl} ; \Omega)$, let us define $\varphi \in \mathrm{H}_{0}^{1}(\Omega)$ the function such that $\left(\varepsilon \nabla \varphi, \nabla \varphi^{\prime}\right)=\left(\varepsilon \boldsymbol{w}, \nabla \varphi^{\prime}\right)$ for all $\varphi^{\prime} \in \mathrm{H}_{0}^{1}(\Omega)$. We have $\boldsymbol{w}-\nabla \varphi \in \mathbf{V}_{N}(\varepsilon ; \Omega)$. Since $\left(\varepsilon \nabla \varphi, \nabla p_{k}\right)=0$, $k=1 \ldots I$, we find $\left(\varepsilon \boldsymbol{w}, \sum_{k=1}^{I} \alpha_{k} \nabla p_{k}\right)=0$.

Since $\mathscr{C}_{0}^{\infty}(\Omega)^{2} \subset \mathbf{H}_{N}(\mathbf{c u r l} ; \Omega)$ is dense in $\mathbf{L}^{2}(\Omega)$, we obtain $\sum_{k=1}^{I} \overline{\alpha_{k}} \nabla p_{k}=0$. But the family $p_{1}, \ldots, p_{I}$ is free. Consequently, we have $\alpha_{1}=\cdots=\alpha_{I}=0$ and the family $\ell_{1}, \ldots, \ell_{I}$ is also free. Theorem 8.4 then allows us to obtain the desired result.

Notations for non simply connected domains. We will assume that there exist connected open surfaces $\Sigma_{j}, j=1 \ldots J$ called "cuts" such that:

i) each surface $\Sigma_{j}$ is an open subset of a smooth variety;

ii) the boundary of $\Sigma_{j}$ is contained in $\partial \Omega, j=1 \ldots J$;

iii) the intersection $\overline{\Sigma_{j}} \cap \overline{\Sigma_{k}}$ is empty for $j \neq k$;

iv) the open set $\dot{\Omega}:=\Omega \backslash \bigcup_{i=1}^{J} \Sigma_{j}$ is pseudo-lipschitz [1] and simply connected. 
The domain $\Omega$ is said topologically trivial when we can take $J=0$. The extension operator from $\mathrm{L}^{2}(\dot{\Omega})$ to $\mathrm{L}^{2}(\Omega)$ is denoted $\sim$ whereas $[\cdot]_{\Sigma_{j}}$ denotes the jump through $\Sigma_{j}, j=1 \ldots J$. In this definition of the jump, we assume that a convention has been established for the sign. We also assume that a unit vector $\boldsymbol{n}$ normal to $\Sigma_{j}, j=1 \ldots J$, is chosen, consistent with the choice of the sign of the jump. Let us introduce the space of scalar potentials

$$
\Theta(\dot{\Omega}):=\left\{\varphi \in \mathrm{H}^{1}(\dot{\Omega}) \mid \int_{\Omega} \widetilde{\varphi}=0 \text { and }[\varphi]_{\Sigma_{j}}=c s t, j=1 \ldots J\right\} .
$$

Let us present a result of decomposition of this space.

Proposition 8.14 Assume $\left(\mathscr{H}_{\mu}\right)$ to be true. Then for $j=1 \ldots J$, there exists a unique solution $q_{j}$ to the problem

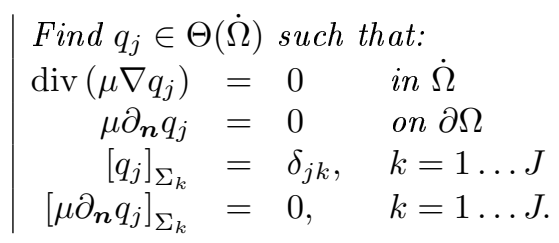

We then have

$$
\Theta(\dot{\Omega})=\mathrm{H}_{\#}^{1}(\Omega) \oplus \operatorname{span}\left(q_{j}\right)_{j=1}^{J} .
$$

Proof. Since we have assumed $\left(\mathscr{H}_{\mu}\right)$ to hold true, problem $(22)$ has at most one solution. Let us build it. For $1 \leq j \leq J$, let $r_{j} \in \Theta(\dot{\Omega})$ be a function such that $\left[r_{j}\right]_{\Sigma_{k}}=\delta_{j k}$ for $k=1 \ldots J$. Then, let us define $q_{j}=r_{j}-\varphi$ where $\varphi$ is the unique element of $\mathrm{H}_{\#}^{1}(\Omega)$ such that

$$
\int_{\Omega} \mu \nabla \varphi \cdot \nabla \varphi^{\prime}=\int_{\Omega} \mu \widetilde{\nabla r_{j}} \cdot \nabla \varphi^{\prime}, \quad \forall \varphi^{\prime} \in \mathrm{H}_{\#}^{1}(\Omega) .
$$

One classically checks that $q_{j}$ satisfies problem (22). This allows to obtain the result of decomposition of the space $\Theta(\dot{\Omega})$.

Let us introduce

$$
\hat{\mathbf{V}}_{T}(\mu ; \Omega):=\{\boldsymbol{u} \in \mathbf{H}(\operatorname{curl} ; \Omega) \mid(\varepsilon \boldsymbol{u}, \widetilde{\nabla \varphi})=0, \forall \varphi \in \Theta(\dot{\Omega})\}
$$

Observe that we have $\hat{\mathbf{V}}_{T}(\mu ; \Omega) \subset \mathbf{V}_{T}(\mu ; \Omega)$. Working as in the proof of Lemma 8.13, we can precise the relation between these two spaces.

Lemma 8.15 Assume $\left(\mathscr{H}_{\mu}\right)$ to be true. For $j=1 \ldots J$, there exists $\boldsymbol{Q}_{j} \in \mathbf{V}_{T}(\mu ; \Omega)$ such that $\left(\mu \boldsymbol{Q}_{j}, \widetilde{\nabla q_{k}}\right)=$ $\delta_{j k}$, for $k=1 \ldots J$. We deduce

$$
\begin{aligned}
\mathbf{V}_{T}(\mu ; \Omega) & =\hat{\mathbf{V}}_{T}(\mu ; \Omega) \oplus \operatorname{span}\left(\boldsymbol{Q}_{j}\right)_{j=1}^{J} \\
\text { and } \quad \mathbf{H}(\operatorname{curl} ; \Omega) & =\hat{\mathbf{V}}_{T}(\mu ; \Omega) \oplus \operatorname{span}\left(\boldsymbol{Q}_{j}\right)_{j=1}^{J} \oplus \nabla \mathrm{H}_{\#}^{1}(\Omega) .
\end{aligned}
$$

Remark 8.16 Theorem 3.12 in [1] states that every element $\boldsymbol{u}$ of $\hat{\mathbf{V}}_{N}(\varepsilon ; \Omega)$ can be written as $\boldsymbol{u}=$ $\varepsilon^{-1} \operatorname{curl} \boldsymbol{\psi}$ with $\boldsymbol{\psi}$ belonging to $\hat{\mathbf{V}}_{T}(1 ; \Omega)$. Similarly, theorem 3.17 of [1] ensures that for every $\boldsymbol{u} \in$ $\hat{\mathbf{V}}_{T}(\mu ; \Omega)$, there exists a unique $\boldsymbol{\psi} \in \hat{\mathbf{V}}_{N}(1 ; \Omega)$ such that $\boldsymbol{u}=\mu^{-1} \mathbf{c u r l} \boldsymbol{\psi}$. In the sequel, we will adapt the proofs of the previous sections using these results of existence of vector potentials.

Assume $\left(\mathscr{H}_{\varepsilon}\right)$ and $\left(\mathscr{H}_{\mu}\right)$ to be true. Remark that Theorems $3.3,3.7$ which prove the equivalence between initial Maxwell's equations and formulations in $\mathbf{V}_{N}(\varepsilon ; \Omega), \mathbf{V}_{T}(\mu ; \Omega)$ do not require any assumption concerning the topology of the domain. Therefore, they are true for the geometry we are considering. In the sequel, we will work with these formulations set in $\mathbf{V}_{N}(\varepsilon ; \Omega), \mathbf{V}_{T}(\mu ; \Omega)$. We should prove the compactness results of Theorems 5.1, 5.3 in the case where $\Omega$ is not simply connected with a non connected boundary. This will be the subject of the next Subsection. 
Remark 8.17 Can we work with formulations set in $\hat{\mathbf{V}}_{N}(\varepsilon ; \Omega), \hat{\mathbf{V}}_{T}(\mu ; \Omega)$ ? A priori, the electric field which satisfies Maxwell's equations has no reason to belong to the space $\hat{\mathbf{V}}_{N}(\varepsilon ; \Omega)$. To see this, we use Lemma 8.13 and we decompose $\boldsymbol{E}$ under the form

$$
\boldsymbol{E}=\hat{\boldsymbol{E}}+\sum_{i=1}^{I} \alpha_{i} \boldsymbol{P}_{i}
$$

with $\hat{\boldsymbol{E}} \in \hat{\mathbf{V}}_{N}(\varepsilon ; \Omega)$ and $\left(\alpha_{1}, \ldots, \alpha_{I}\right) \in \mathbb{C}^{I}$. For $i=1 \ldots I$, testing with $\nabla p_{i}$ in (6), we find

$$
\alpha_{i}=\left(\varepsilon \boldsymbol{E}, \nabla p_{i}\right)=(i \omega)^{-1}\left(\boldsymbol{J}, \nabla p_{i}\right)=(i \omega)^{-1}\langle\boldsymbol{J} \cdot \boldsymbol{n}, 1\rangle_{\Gamma_{i}},
$$

where $\langle\cdot, \cdot\rangle_{\Gamma_{i}}$ denotes the duality product between $\mathrm{H}^{1 / 2}\left(\Gamma_{i}\right)$ and its dual space. Above, we have used the properties $\operatorname{div} \boldsymbol{J}=0$ in $\Omega$ and $p_{i}=\delta_{i k}$ on $\Gamma_{k}, k=1 \ldots I$. Thus, if there exists $0 \leq i \leq I$ such that $\langle\boldsymbol{J} \cdot \boldsymbol{n}, 1\rangle_{\Gamma_{i}} \neq 0$, then $\boldsymbol{E}$ does not belong to $\hat{\mathbf{V}}_{N}(\varepsilon ; \Omega)$. But this also proves that to know the field $\boldsymbol{E}$, it is sufficient to determine $\hat{\boldsymbol{E}}$. Following the same reasoning, we can check that the magnetic field is always an element of $\hat{\mathbf{V}}_{T}(\mu ; \Omega)$, regardless of the source term $\boldsymbol{J}$.

\subsubsection{Non trivial geometry: compactness results}

Let us work first on the space of electric fields.

Theorem 8.18 Let $\Omega$ be a bounded connected open subset of $\mathbb{R}^{3}$ with a Lipschitz-continuous boundary. Assume $\left(\mathscr{H}_{\varepsilon}\right)$ to be true. Then the embedding of $\mathbf{V}_{N}(\varepsilon ; \Omega)$ in $\mathbf{L}^{2}(\Omega)$ is compact.

Proof. Let $\left(\boldsymbol{u}_{n}\right)$ be a bounded sequence of $\mathbf{V}_{N}(\varepsilon ; \Omega)$. For all $n \in \mathbb{N}$, using Lemma 8.13, let us define $\boldsymbol{v}_{n} \in \hat{\mathbf{V}}_{N}(\varepsilon ; \Omega)$ and $\left(\alpha_{1 n}, \ldots, \alpha_{I n}\right) \in \mathbb{C}^{I}$ the elements such that $\boldsymbol{u}_{n}=\boldsymbol{v}_{n}+\sum_{i=1}^{I} \alpha_{i n} \boldsymbol{P}_{i}$. To prove Theorem 8.18, it is sufficient to show that we can extract a subsequence from $\left(\boldsymbol{v}_{n}\right)$ which converges in $\mathbf{L}^{2}(\Omega)$. Define $\boldsymbol{F}_{n}=\operatorname{curl} \boldsymbol{v}_{n}$. The sequence $\left(\boldsymbol{F}_{n}\right)$ is bounded in $\mathbf{L}^{2}(\Omega)$. In addition, for all $n \in \mathbb{N}$, there holds $\left(\varepsilon \boldsymbol{v}_{n}, \nabla \varphi\right)=0$ for all $\varphi \in \mathrm{H}_{\Gamma}^{1}(\Omega)$. Therefore, according to theorem 3.12 in [1], there exists $\boldsymbol{w}_{n} \in \hat{\mathbf{V}}_{T}(1 ; \Omega)$ such that $\operatorname{curl} \boldsymbol{w}_{n}=\varepsilon \boldsymbol{v}_{n}$. Thus, for all $n \in \mathbb{N}$, we have $\boldsymbol{v}_{n}=\varepsilon^{-1} \mathbf{c u r l} \boldsymbol{w}_{n}$. Let us prove that we can extract a subsequence from $\left(\operatorname{curl} \boldsymbol{w}_{n}\right)$ which converges in $\mathbf{L}^{2}(\Omega)$.

We know that $\boldsymbol{w} \mapsto\|\operatorname{curl} \boldsymbol{w}\|$ defines a norm on $\hat{\mathbf{V}}_{T}(1 ; \Omega)$ (cf. [1, corollary 3.16]). Consequently, the sequence $\left(\boldsymbol{w}_{n}\right)$ is bounded in $\hat{\mathbf{V}}_{T}(1 ; \Omega)$. Since $\operatorname{curl} \varepsilon^{-1} \operatorname{curl} \boldsymbol{w}_{n}=\boldsymbol{F}_{n}$ in $\Omega$ and $\left(\varepsilon^{-1} \operatorname{curl} \boldsymbol{w}_{n}\right) \times \boldsymbol{n}=0$ on $\partial \Omega$, we have $\left(\varepsilon^{-1} \operatorname{curl} \boldsymbol{w}_{n}, \operatorname{curl} \boldsymbol{w}^{\prime}\right)=\left(\boldsymbol{F}_{n}, \boldsymbol{w}^{\prime}\right)$ for all $\boldsymbol{w}^{\prime} \in \hat{\mathbf{V}}_{T}(1 ; \Omega)$.

Now, let us build a bounded operator $\hat{\mathbb{T}}$ from $\hat{\mathbf{V}}_{T}(1 ; \Omega)$ to $\hat{\mathbf{V}}_{T}(1 ; \Omega)$ to recover some positivity. Consider $\boldsymbol{w} \in \hat{\mathbf{V}}_{T}(1 ; \Omega)$.

i) First, define $\varphi$ the unique element of $\mathrm{H}_{0}^{1}(\Omega)$ such that

$$
\int_{\Omega} \varepsilon \nabla \varphi \cdot \nabla \varphi^{\prime}=\int_{\Omega} \varepsilon \operatorname{curl} \boldsymbol{w} \cdot \nabla \varphi^{\prime}, \quad \forall \varphi^{\prime} \in \mathrm{H}_{0}^{1}(\Omega)
$$

The function $\varphi$ is well-defined because we have assumed $\left(\mathscr{H}_{\varepsilon}\right)$ to be true.

ii) Defining $\beta_{i}:=\left(\varepsilon \operatorname{curl} \boldsymbol{w}, \nabla p_{i}\right)$ for $i=1 \ldots I$, let us then notice that we have $\left(\varepsilon\left(\operatorname{curl} \boldsymbol{w}-\sum_{i=1}^{I} \beta_{i} \boldsymbol{P}_{i}-\right.\right.$ $\left.\nabla \varphi), \nabla \varphi^{\prime}\right)=0$ for all $\varphi^{\prime} \in \mathrm{H}_{\Gamma}^{1}(\Omega)$. Consequently, according to theorem 3.12 of [1], there exists a unique potential $\hat{\mathbb{T}} \boldsymbol{w} \in \hat{\mathbf{V}}_{T}(1 ; \Omega)$ such that $\operatorname{curl} \hat{\mathbb{T}} \boldsymbol{w}=\varepsilon\left(\operatorname{curl} \boldsymbol{w}-\sum_{i=1}^{I} \beta_{i} \boldsymbol{P}_{i}-\nabla \varphi\right)$. This defines a bounded operator $\hat{\mathbb{T}}$ from $\hat{\mathbf{V}}_{T}(1 ; \Omega)$ to $\hat{\mathbf{V}}_{T}(1 ; \Omega)$.

Since $\hat{\mathbb{T}}$ is continuous, the sequence $\left(\hat{\mathbb{T}} \boldsymbol{w}_{n}\right)$ is bounded in $\hat{\mathbf{V}}_{T}(1 ; \Omega)$. So we can extract a subsequence from $\left(\boldsymbol{w}_{n}\right)$, still denoted $\left(\boldsymbol{w}_{n}\right)$, such that $\left(\hat{\mathbb{T}} \boldsymbol{w}_{n}\right)$ converges in $\mathbf{L}^{2}(\Omega)$. Since for $i=1 \ldots I$, the sequence $\left(\beta_{\text {in }}\right)$, with $\beta_{\text {in }}=\left(\varepsilon \operatorname{curl} \boldsymbol{w}_{n}, \nabla p_{i}\right)$, is bounded in $\mathbb{C}$, we can extract a subsequence from $\left(\boldsymbol{w}_{n}\right)$, still denoted $\left(\boldsymbol{w}_{n}\right)$, such that $\left(\beta_{i n}\right)$ converges. Let us introduce $\boldsymbol{w}_{n m}:=\boldsymbol{w}_{n}-\boldsymbol{w}_{m}, \boldsymbol{F}_{n m}:=\boldsymbol{F}_{n}-\boldsymbol{F}_{m}$ and $\beta_{\text {inm }}:=\beta_{\text {in }}-\beta_{\text {im }}$. We have

$$
\begin{aligned}
\left|\left(\boldsymbol{F}_{n m}, \hat{\mathbb{T}} \boldsymbol{w}_{n m}\right)\right| & =\left|\left(\varepsilon^{-1} \operatorname{curl} \boldsymbol{w}_{n m}, \operatorname{curl} \hat{\mathbb{T}} \boldsymbol{w}_{n m}\right)\right| \\
& \geq\left\|\operatorname{curl} \boldsymbol{w}_{n m}\right\|^{2}-\sum_{i=1}^{I}\left|\beta_{i n m} \|\left(\operatorname{curl} \boldsymbol{w}_{n m}, \boldsymbol{P}_{i}\right)\right|
\end{aligned}
$$

This estimate proves that $\left(\operatorname{curl} \boldsymbol{w}_{n}\right)$ is a Cauchy sequence of $\mathbf{L}^{2}(\Omega)$. Therefore, it converges. Thus, we can extract a subsequence from $\left(\boldsymbol{v}_{n}\right)=\left(\varepsilon^{-1} \operatorname{curl} \boldsymbol{w}_{n}\right)$ which converges in $\mathbf{L}^{2}(\Omega)$. 
Proceeding in the same way, we prove the

Theorem 8.19 Let $\Omega$ be a bounded connected open subset of $\mathbb{R}^{3}$ with a Lipschitz-continuous boundary. Assume $\left(\mathscr{H}_{\mu}\right)$ to be true. Then the embedding of $\mathbf{V}_{T}(\mu ; \Omega)$ in $\mathbf{L}^{2}(\Omega)$ is compact.

\subsubsection{Non trivial geometry: well-posedness of Maxwell's equations}

Let us state now the main result of this section concerning the well-posedness of Maxwell's equations in non trivial geometries.

Theorem 8.20 Let $\Omega$ be a bounded connected open subset of $\mathbb{R}^{3}$ with a Lipschitz-continuous boundary. Consider $\boldsymbol{J} \in \mathbf{L}^{2}(\Omega)$ such that $\operatorname{div} \boldsymbol{J}=0$. Assume $\left(\mathscr{H}_{\varepsilon}\right)$ and $\left(\mathscr{H}_{\mu}\right)$ to be true. Then, we have the following results.

- For all $\omega \in \mathbb{C}$, the operator for the electric field $\mathscr{A}_{N}(\omega): \mathbf{V}_{N}(\varepsilon ; \Omega) \rightarrow \mathbf{V}_{N}(\varepsilon ; \Omega)$ defined in (8) is a Fredholm operator of index 0 . Moreover, for $\omega \in \mathbb{C}^{*}, \boldsymbol{E} \in \mathbf{V}_{N}(\varepsilon ; \Omega)$ satisfies $\left(\mathscr{A}_{N}(\omega) \boldsymbol{E}, \boldsymbol{E}^{\prime}\right)_{\text {curl }}=$ $i \omega\left(\boldsymbol{J}, \boldsymbol{E}^{\prime}\right)$, for all $\boldsymbol{E}^{\prime} \in \mathbf{V}_{N}(\varepsilon ; \Omega)$, if and only if the pair $\left(\boldsymbol{E},(i \omega \mu)^{-1} \mathbf{c u r l} \boldsymbol{E}\right)$ satisfies the Maxwell's equations (1)-(2).

- For all $\omega \in \mathbb{C}, \mathscr{A}_{T}(\omega): \mathbf{V}_{T}(\mu ; \Omega) \rightarrow \mathbf{V}_{T}(\mu ; \Omega)$ defined in (11) is a Fredholm operator of index 0 . Moreover, for $\omega \in \mathbb{C}^{*}, \boldsymbol{H} \in \mathbf{V}_{T}(\mu ; \Omega)$ satisfies $\left(\mathscr{A}_{T}(\omega) \boldsymbol{H}, \boldsymbol{H}^{\prime}\right)_{\mathbf{c u r l}}=\left(\varepsilon^{-1} \boldsymbol{J}, \mathbf{c u r l} \boldsymbol{H}^{\prime}\right)$, for all $\boldsymbol{H}^{\prime} \in$ $\mathbf{V}_{T}(\mu ; \Omega)$, if and only if the pair $\left(i(\omega \varepsilon)^{-1}(\mathbf{c u r l} \boldsymbol{H}-\boldsymbol{J}), \boldsymbol{H}\right)$ satisfies the Maxwell's equations (1)-(2).

Proof. Let us prove that $\mathscr{A}_{N}(\omega)$ is a Fredholm operator of index 0. For all $\omega \in \mathbb{C}$, using Theorem 8.18, one can prove that $\mathscr{A}_{N}(\omega)-\mathscr{A}_{N}(0)$ is a compact operator of $\mathbf{V}_{N}(\varepsilon ; \Omega)$. So, it is sufficient to show that $\mathscr{A}_{N}(0)$ is Fredholm of index 0 . Again, we are going to build a right parametrix $\mathbb{T}^{\varepsilon}: \mathbf{V}_{N}(\varepsilon ; \Omega) \rightarrow \mathbf{V}_{N}(\varepsilon ; \Omega)$ for the operator $\mathscr{A}_{N}(0)$. Consider $\boldsymbol{u} \in \mathbf{V}_{N}(\varepsilon ; \Omega)$.

i) First, define $\varphi$ the unique element of $\mathrm{H}_{\#}^{1}(\Omega)$ such that

$$
\int_{\Omega} \mu \nabla \varphi \cdot \nabla \varphi^{\prime}=\int_{\Omega} \mu \operatorname{curl} \boldsymbol{u} \cdot \nabla \varphi^{\prime}, \quad \forall \varphi^{\prime} \in \mathrm{H}_{\#}^{1}(\Omega) .
$$

The function $\varphi$ is well-defined because we have assumed $\left(\mathscr{H}_{\mu}\right)$ to hold true.

ii) Defining $\beta_{j}:=\left(\mu \operatorname{curl} \boldsymbol{w}, \widetilde{\nabla q_{j}}\right)$ for $j=1 \ldots J$, notice that we have $\left(\mu\left(\operatorname{curl} \boldsymbol{w}-\sum_{j=1}^{J} \beta_{j} \boldsymbol{Q}_{j}-\right.\right.$ $\left.\nabla \varphi), \widetilde{\nabla \varphi^{\prime}}\right)=0$ for all $\varphi^{\prime} \in \Theta(\dot{\Omega})$. Therefore, according to theorem 3.17 in [1], there exists a unique potential $\boldsymbol{\psi} \in \hat{\mathbf{V}}_{N}(1 ; \Omega)$ such that $\operatorname{curl} \boldsymbol{\psi}=\mu\left(\operatorname{curl} \boldsymbol{w}-\sum_{j=1}^{J} \beta_{j} \boldsymbol{Q}_{j}-\nabla \varphi\right)$.

iii) Consider $\zeta$ the unique element of $\mathrm{H}_{0}^{1}(\Omega)$ such that

$$
\int_{\Omega} \varepsilon \nabla \zeta \cdot \nabla \zeta^{\prime}=\int_{\Omega} \varepsilon \psi \cdot \nabla \zeta^{\prime}, \quad \forall \zeta^{\prime} \in \mathrm{H}_{0}^{1}(\Omega) .
$$

The function $\zeta$ is well-defined because we have assumed $\left(\mathscr{H}_{\varepsilon}\right)$ to hold true.

iv) Finally, let us define the operator $\mathbb{T}^{\varepsilon}: \mathbf{V}_{N}(\varepsilon ; \Omega) \rightarrow \mathbf{V}_{N}(\varepsilon ; \Omega)$ such that $\mathbb{T}^{\varepsilon} \boldsymbol{u}=\boldsymbol{\psi}-\nabla \zeta$ and the operator $K^{\varepsilon}: \mathbf{V}_{N}(\varepsilon ; \Omega) \rightarrow \mathbf{V}_{N}(\varepsilon ; \Omega)$ such that

$$
\left(K^{\varepsilon} \boldsymbol{u}, \boldsymbol{v}\right)_{\operatorname{curl}}=(\boldsymbol{u}, \boldsymbol{v})+\sum_{j=1}^{J}\left(\mu \operatorname{curl} \boldsymbol{u}, \widetilde{\nabla q_{j}}\right)\left(\boldsymbol{Q}_{j}, \operatorname{curl} \boldsymbol{v}\right), \quad \forall \boldsymbol{v} \in \mathbf{V}_{N}(\varepsilon ; \Omega) .
$$

According to Theorem 8.18, we know that the embedding of $\mathbf{V}_{N}(\varepsilon ; \Omega)$ in $\mathbf{L}^{2}(\Omega)$ is compact. Consequently, $K^{\varepsilon}$ is the sum of a compact operator and a finite rank operator. Therefore, it is a compact operator. Now, for all $\boldsymbol{v} \in \mathbf{V}_{N}(\varepsilon ; \Omega)$, we find

$$
\begin{aligned}
\left(\mathscr{A}_{N}(0)\left(\mathbb{T}^{\varepsilon} \boldsymbol{u}\right), \boldsymbol{v}\right)_{\operatorname{curl}} & =\left(\mu^{-1} \operatorname{curl}\left(\mathbb{T}^{\varepsilon} \boldsymbol{u}\right), \operatorname{curl} \boldsymbol{v}\right) \\
& =(\operatorname{curl} \boldsymbol{u}, \operatorname{curl} \boldsymbol{v})+(\boldsymbol{u}, \boldsymbol{v})-\left(K^{\varepsilon} \boldsymbol{u}, \boldsymbol{v}\right)_{\text {curl }}
\end{aligned}
$$

Thus, we have $\mathscr{A}_{T}(0) \circ \mathbb{T}^{\varepsilon}+K^{\varepsilon}=\mathrm{Id}$. We deduce that $\mathbb{T}^{\varepsilon}$ is indeed a right parametrix for $\mathscr{A}_{N}(0)$. This proves that the selfadjoint operator $\mathscr{A}_{N}(0)$ is Fredholm of index 0. Similarly, we prove that $\mathscr{A}_{T}(\omega)$ : $\mathbf{V}_{T}(\mu ; \Omega) \rightarrow \mathbf{V}_{T}(\mu ; \Omega)$ is a Fredholm operator of index 0 for all $\omega \in \mathbb{C}$. Finally, the equivalence with Maxwell's equations (1)-(2) comes from Theorems 3.3 and 3.7. 
Remark 8.21 Again, to apply the analytic Fredholm theorem to prove that Maxwell's equations (1)-(2) are uniquely solvable for all $\omega \in \mathbb{C}^{*} \backslash S$ where $S$ is a discrete set, it remains to show that there exists $\omega \in \mathbb{C}$ such that $\mathscr{A}_{N}(\omega)$ or $\mathscr{A}_{T}(\omega)$ is invertible. This results does not seem simple to obtain. However, according to Remark 8.17, we observe that the elements of ker $\mathscr{A}_{N}(\omega)$ (resp. ker $\mathscr{A}_{T}(\omega)$ ) always belong to $\hat{\mathbf{V}}_{N}(\varepsilon ; \Omega)$ (resp. $\hat{\mathbf{V}}_{T}(\mu ; \Omega)$ ). On the other hand, the map $\boldsymbol{u} \mapsto\|\mathbf{c u r l} \boldsymbol{u}\|$ defines a norm on $\hat{\mathbf{V}}_{N}(\varepsilon ; \Omega)$ and on $\hat{\mathbf{V}}_{T}(\mu ; \Omega)$. But one ingredient is still missing to achieve the injectivity of $\mathscr{A}_{N}(\omega)$ or $\mathscr{A}_{T}(\omega)$.

\section{Acknowledgments}

This work was supported by the ANR project METAMATH, grant ANR-11-MONU-016 of the French Agence Nationale de la Recherche.

\section{References}

[1] C. Amrouche, C. Bernardi, M. Dauge, and V. Girault. Vector potentials in three-dimensional nonsmooth domains. Math. Meth. Appl. Sci., 21:823-864, 1998.

[2] A.-S. Bonnet-Ben Dhia, L. Chesnel, and P. Ciarlet Jr. T-coercivity for scalar interface problems between dielectrics and metamaterials. Math. Mod. Num. Anal., 46:1363-1387, 2012.

[3] A.-S. Bonnet-Ben Dhia, L. Chesnel, and P. Ciarlet Jr. Two-dimensional Maxwell's equations with sign-changing coefficients. Submitted, Special Issue: NELIA 2011, 2012.

[4] A.-S. Bonnet-Ben Dhia, L. Chesnel, and X. Claeys. Radiation condition for a non-smooth interface between a dielectric and a metamaterial. Accepted in Math. Models Meth. App. Sci., 2012.

[5] A.-S. Bonnet-Ben Dhia, P. Ciarlet Jr., and C.M. Zwölf. Two-and three-field formulations for wave transmission between media with opposite sign dielectric constants. J. of Comput. and Appl. Math., 204(2):408-417, 2007.

[6] A.-S. Bonnet-Ben Dhia, P. Ciarlet Jr., and C.M. Zwölf. A new compactness result for electromagnetic waves. Application to the transmission problem between dielectrics and metamaterials. Math. Models Meth. App. Sci., 18:1605-1631, 2008.

[7] A.-S. Bonnet-Ben Dhia, P. Ciarlet Jr., and C.M. Zwölf. Time harmonic wave diffraction problems in materials with sign-shifting coefficients. J. Comput. Appl. Math, 234:1912-1919, 2010. Corrigendum J. Comput. Appl. Math., 234:2616, 2010.

[8] A.-S. Bonnet-Ben Dhia, D. Dauge, and K. Ramdani. Analyse spectrale et singularités d'un problème de transmission non coercif. C. R. Acad. Sci. Paris, Ser. I, 328:717-720, 1999.

[9] M. Cessenat. Mathematical methods in electromagnetism: linear theory and applications. Series on advances in mathematics for applied sciences. World Scientific, 1996.

[10] L. Chesnel. Investigation of some transmission problems with sign-changing coefficients. Application to metamaterials. PhD thesis, École Polytechnique, 2012.

[11] L. Chesnel and P. Ciarlet Jr. Compact imbeddings in electromagnetism with interfaces between classical materials and metamaterials. SIAM J. on Mathematical Analysis, 43(5):2150-2169, 2011.

[12] L. Chesnel and P. Ciarlet Jr. T-coercivity and continuous Galerkin methods: application to transmission problems with sign changing coefficients. Accepted in Numer. Math., 2012.

[13] E. T. Chung and P. Ciarlet Jr. A staggered discontinuous Galerkin method for wave propagation in media with dielectrics and meta-materials. Journal of Computational and Applied Mathematics, 239:189-207, 2013.

[14] M. Costabel and E. Stephan. A direct boundary integral method for transmission problems. J. of Math. Anal. and Appl., 106:367-413, 1985. 
[15] M. Dauge and B. Texier. Problèmes de transmission non coercifs dans des polygones. Technical Report 97-27, Université de Rennes 1, IRMAR, Campus de Beaulieu, 35042 Rennes Cedex, France, 1997. http://hal.archives-ouvertes.fr/docs/00/56/23/29/PDF/BenjaminT_arxiv.pdf.

[16] N. Engheta. An idea for thin subwavelength cavity resonators using metamaterials with negative permittivity and permeability. Antennas and Wireless Propagation Letters, IEEE, 1:10-13, 2002.

[17] P. Fernandes and M. Raffetto. Well posedness and finite element approximability of time-harmonic electromagnetic boundary value problems involving bianisotropic materials and metamaterials. Math. Models Meth. App. Sci., 19(12):2299-2335, 2009.

[18] V. Girault and P.-A. Raviart. Finite element methods for Navier-Stokes equations, volume 5 of Springer Series in Computational Mathematics. Springer-Verlag, Berlin, 1986.

[19] C. Hazard and M. Lenoir. On the solution of time-harmonic scattering problems for Maxwell's equations. SIAM J. Math. Anal., 27:1597-1630, 1996.

[20] R. Kress. Linear integral equations, Second Edition, volume 82 of Applied Mathematical Sciences. Springer Verlag, 1999.

[21] D. Maystre and S. Enoch. Perfect lenses made with left-handed materials: Alice's mirror? JOSA A, 21(1):122-131, 2004.

[22] W. McLean. Strongly elliptic systems and boundary integral equations. Cambridge University Press, Cambridge, 2000.

[23] P. Monk. Finite element methods for Maxwell's equations. Numerical Mathematics and Scientific Computation. Oxford University Press, New York, 2003.

[24] S. Nicaise and J. Venel. A posteriori error estimates for a finite element approximation of transmission problems with sign changing coefficients. Journal of Computational and Applied Mathematics, 235(14):4272-4282, 2011.

[25] P. Ola. Remarks on a transmission problem. J. Math. Anal. Appl., 196:639-658, 1995.

[26] G. Oliveri and M. Raffetto. A warning about metamaterials for users of frequency-domain numerical simulators. Antennas and Propagation, IEEE Transactions on, 56(3):792-798, 2008.

[27] J.B. Pendry. Negative refraction makes a perfect lens. Physical Review Letters, 85(18):3966-3969, 2000.

[28] M. Raffetto. Ill-posed waveguide discontinuity problem involving metamaterials with impedance boundary conditions on the two ports. Science, Measurement \& Technology, IET, 1(5):232-239, 2007.

[29] K. Ramdani. Lignes supraconductrices: analyse mathématique et numérique. PhD thesis, Université Paris 6, 1999.

[30] A.A. Sukhorukov, I.V. Shadrivov, and Y.S. Kivshar. Wave scattering by metamaterial wedges and interfaces. Int. J. Numer. Model., 19:105-117, 2006.

[31] V.G. Veselago. The electrodynamics of substances with simultaneously negative values of $\varepsilon$ and $\mu$. Sov. Phys. Usp, 10:509-514, 1968.

[32] H. Wallén, H. Kettunen, and A. Sihvola. Surface modes of negative-parameter interfaces and the importance of rounding sharp corners. Metamaterials, 2(2-3):113-121.

[33] C. Weber. A local compactness theorem for Maxwell's equations. Math. Meth. Appl. Sci., 2:12-25, 1980 .

[34] J. Wloka. Partial Differential Equations. Cambridge Univ. Press, 1987.

[35] C.M. Zwölf. Méthodes variationnelles pour la modélisation des problèmes de transmission d'onde électromagnétique entre diélectrique et méta-matériau. $\mathrm{PhD}$ thesis, Université Versailles - SaintQuentin, 2008. 